Trans

continentales
Transcontinentales

Sociétés, idéologies, système mondial

$4 \mid 2007$

Amérique latine

\title{
Territoires, sociétés et mondialisation en Amérique andine
}

Territories, societies and globalisation in Andean America

Jean-Paul Deler et Henri R. Godard

\section{(2) OpenEdition}

\section{Journals}

Édition électronique

URL : http://journals.openedition.org/transcontinentales/581

DOI : 10.4000/transcontinentales.581

ISBN : 978-2-7351-1565-5

ISSN : $1775-397 X$

\section{Éditeur}

Editions de la maison des sciences de l'homme

\section{Édition imprimée}

Date de publication : 1 juin 2007

Pagination : 15-44

ISBN : 978-2-200-92396-9

ISSN : $1950-1684$

\section{Référence électronique}

Jean-Paul Deler et Henri R. Godard, «Territoires, sociétés et mondialisation en Amérique andine »

Transcontinentales [En ligne], 4| 2007, document 2, mis en ligne le 27 avril 2011, consulté le 08

septembre 2020. URL : http://journals.openedition.org/transcontinentales/581 ; DOI : https://doi.org/ 10.4000/transcontinentales.581 


\title{
Territoires, sociétés et mondialisation en Amérique andine
}

\author{
Jean-Paul Deler et Henri R. Godard
}

Vastes espaces marqués par la présence de la plus grande montagne du monde et ses effets sur les disparités de l'occupation humaine; héritages partagés d'une longue histoire commune, de la mise en place du peuplement à l'évolution des «modèles» d'exploitation économiques des territoires pendant les périodes coloniale et républicaine; prégnance d'une économie tournée vers l'extérieur; mouvement continu d'urbanisation et de métropolisation; maintien des inégalités spatiales et socioéconomiques; parenté relative des parcours politiques des quatre dernières décennies (des dictatures et régimes autoritaires à la transition démocratique et au retour des "gauches» au pouvoir); reconnaissance officielle du multiculturalisme des sociétés... Voici quelques-unes des clés de lecture pour évaluer la communauté de destin de l'ensemble de l'Amérique latine, vaste ensemble de 570 millions d'habitants et de 23 millions de $\mathrm{km}^{2}$ (l'Union européenne des 27 compte 494 millions $\mathrm{d}^{\prime}$ habitants et 4,4 millions de $\mathrm{km}^{2}$ ). Qu'il s'agisse des textes fondateurs de la fin du $\mathrm{XIX}^{\mathrm{e}}$ siècle et de la première moitié $\mathrm{du} \mathrm{Xx}^{\mathrm{e}}$, $\mathrm{d}^{\prime}$ ouvrages de référence comme le volume Amérique latine de la Géographie universelle (1991) ou des derniers livres publiés en 2005 et 2006, tous les auteurs ${ }^{1}$ mettent l'accent sur les facteurs historiques, sociaux et économiques, les caractéristiques physiques et les modes de structuration de l'espace qui donnent à ce "continent» une homogénéité certaine. Cependant, à changer d'échelle de travail, ils mettent aussi en évidence la diversité des milieux et des sociétés et les profondes différences régionales, nationales ou infranationales.

Le choix proposé ici est d'aborder l'un des sous-ensembles de l'espace latinoaméricain, le monde andin, à travers ses États, ses populations et son organisation socioéconomique, le maillage de ses territoires et ses dynamiques spatiales. Un sousensemble dont la cordillère des Andes constitue un élément majeur de structuration

1 - Voir bibliographie en fin d'article.

Jean-Paul Deler : Géographe, directeur de recherche au CNRS.

Henri R. Godard : Directeur de l'Institut français d'études andines (IFÉA). 
de l'espace susceptible de donner une part de son unité à cette région du monde qui rassemble sept États couvrant une superficie de plus de 8 millions de $\mathrm{km}^{2}$ et peuplés de 180 millions d'habitants; soit un peu plus de la moitié de la superficie et de la population de l'Amérique du Sud (fig. 1 et 2). Dans le contexte contemporain de mondialisation, d'internationalisation des échanges et aussi de crises et de recompositions des marchés régionaux de l'Amérique du Sud, il s'agit ici de questionner et d'évaluer, à différentes échelles d'espace et de temps, les éléments d'unité et de différenciation, les solidarités construites et les divergences d'intérêts dans ce groupe de sept pays. Les Andes imposent leur marque, jamais superficielle, à l'ensemble de cet espace, mais elle n'est pas indifférenciée : certains États sont profondément andins et d'autres le sont moins. Et si au niveau sous-continental, l'Amérique andine offre le visage d'une relative homogénéité, du fait de tendances lourdes qui demeurent (organisation économique, structuration des espaces), à un niveau plus fin d'analyse, ruptures, discontinuités et dissymétries apparaissent et les disparités se font jour.

\section{Un grand espace du monde}

La cordillère des Andes, la plus grande chaîne de montagne du monde, s'étend du nord au sud du sous-continent sur $7500 \mathrm{~km}$ et couvre plus de $65^{\circ}$ de latitude, de $11^{\circ} \mathrm{N}$ à $55^{\circ} \mathrm{S}$. On y observe la plus importante dénivellation de l'écorce terrestre, entre la fosse océanique d'Atacama (profonde de $8000 \mathrm{~m}$ ) et des sommets voisins à près de $6000 \mathrm{~m}$. Elle borde au nord le bassin amazonien qui couvre une superficie de plus de 7 millions de $\mathrm{km}^{2}$. Cette barrière continue, marquée par l'intensité de l'activité sismique et volcanique («ceinture de feu du Pacifique»), joue un rôle essentiel dans la répartition zonale des climats. Massive et large dans sa partie centrale (plus de $500 \mathrm{~km}$ en Bolivie), elle est plus étroite au nord et au sud (moins d'une centaine de kilomètres). Si le point culminant des Amériques et de l'hémisphère sud est situé dans le sud de la cordillère - l'Aconcagua, en Argentine, atteint $6959 \mathrm{~m} \mathrm{-,} \mathrm{la} \mathrm{plupart} \mathrm{de} \mathrm{ses} \mathrm{plus} \mathrm{hauts} \mathrm{sommets,} \mathrm{compris} \mathrm{entre} 5000$ et plus de 6000 m, sont concentrés dans la partie médiane de la chaîne; à ses extrémités nord et sud, le relief s'affaiblit considérablement, dans la cordillère caraïbe, le long du littoral vénézuélien, et dans la cordillère de Magellan, en Terre de Feu (fig. 1). La cordillère, par son extension en latitude, relève d'une gamme très variée de climats et de milieux (du tropical au subpolaire), les variations d'altitude et de volume du relief induisant d'importantes variantes et nuances bioclimatiques, aux niveaux régional ou local... Ces contraintes naturelles, présentes dans tous les États andins, jouent un rôle majeur dans la structuration et l'organisation de l'espace, mais leur impact sur les compositions territoriales est plus ou moins marqué selon les pays.

Cette puissante colonne vertébrale physique sud-américaine, ne saurait pour autant rendre compte à elle seule des formes d'occupation de l'espace, de répartition du peuplement et de mise en valeur ou des dynamiques économiques et territoriales.

\section{Continuité orographique, gradients et dissymétries bioclimatiques}

Située, pour l'essentiel, en bordure pacifique du sous-continent, la barrière andine se développe sur environ 2,5 millions de $\mathrm{km}^{2}$ - à peine un tiers de la superficie de 


\section{Figure 1 : Pleins et «VIDes» relatifs ou QuASI absolus}

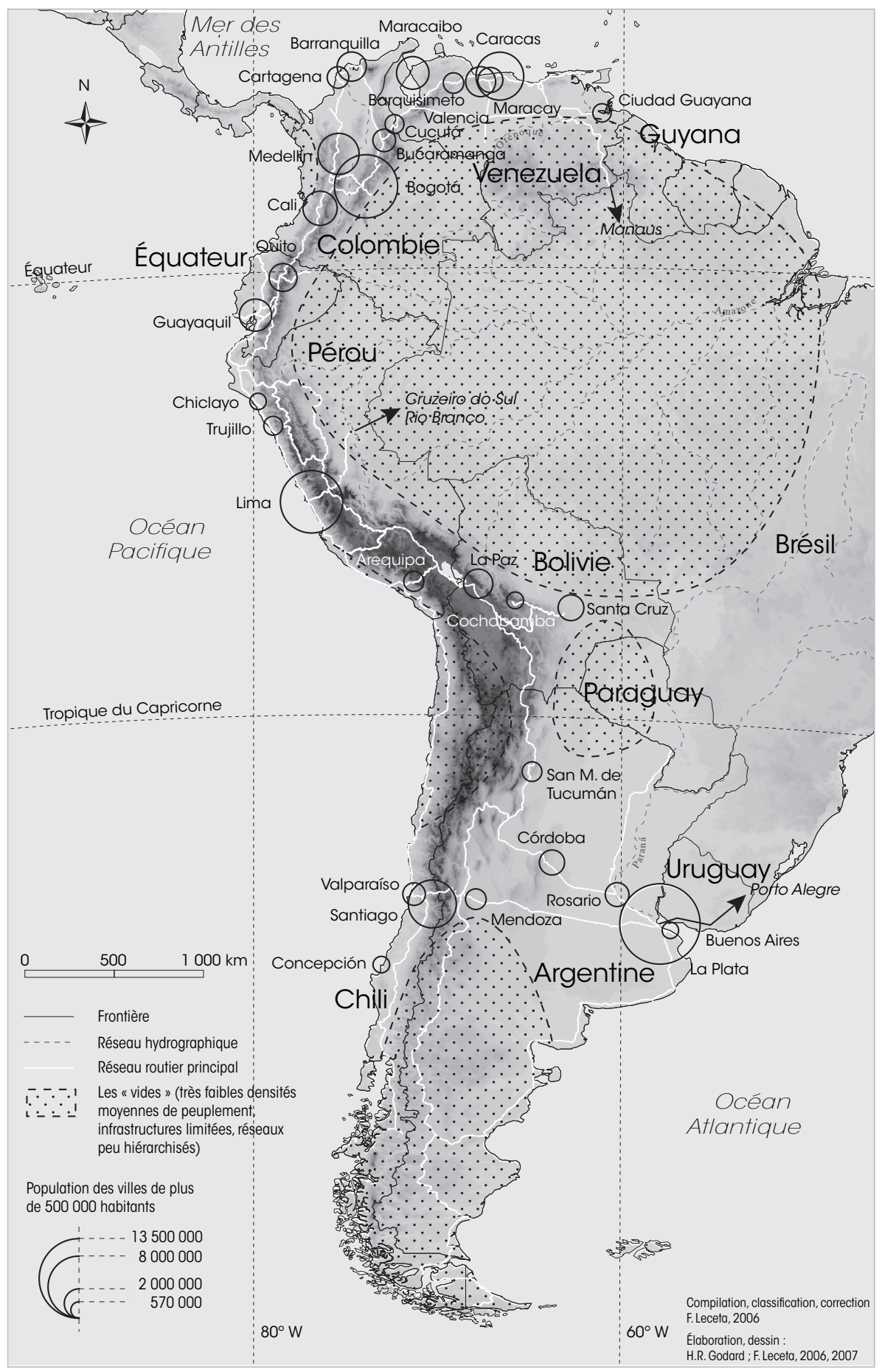

Sources : ESRI Data \& Maps, 2004 ; University of California-Berkeley (modèle numérque de terrain) ; GeoHyve, 2007 (pop.) 
l'Amérique du Sud - dont 1,1 million de $\mathrm{km}^{2}$ situés à plus de $2500 \mathrm{~m}$ d'altitude; elle imprime sa marque, d'ampleur inégale, dans sept pays.

Schématiquement, trois ensembles méridiens s'alignent de l'océan Pacifique à l'intérieur du continent. À l'ouest, le piémont littoral, qui est assez large de la Colombie au nord du Pérou (plus de $150 \mathrm{~km}$ en Équateur), n'est qu'une bande étroite ou un simple liseré plus au sud. Cet espace côtier est dominé par le massif montagneux proprement dit; la cordillère, unique entre Chili et Argentine, se complexifie vers le nord où elle se divise en deux chaînes parallèles, voire trois, notamment en Colombie. Des bassins, des vallées et des plateaux sont enchâssés entre les chaînes : ainsi la succession des bassins d'Équateur entre deux branches de la cordillère séparées seulement d'une cinquantaine de kilomètres, ou le vaste altiplano qui s'étend du sud du Pérou au nord de l'Argentine et du Chili avec ses lacs (Titicaca) et ses vastes étendues de sel (salares d'Uyuni et d'Atacama). D'importantes ressources métallifères (or, argent, étain, cuivre, plomb...) sont concentrées dans ces hautes terres; utilisées depuis la période précolombienne et objet de toutes les convoitises lors de la conquête espagnole, leur exploitation coloniale est à l'origine d'une déstructuration des espaces, des économies et des sociétés locales ; du Pérou au Chili, les gisements miniers sont aujourd'hui intensément exploités, souvent par des compagnies transnationales. Enfin, à l'est de la cordillère des Andes s'étend un vaste piémont de terres basses, domaine des grands fleuves de la cuvette amazonienne, de la Colombie à la Bolivie, et du système hydrographique du Paraná (plaine intérieure des pampas argentines).

FIGURE 2 : DONNÉES DE CADRAGE

\begin{tabular}{|r|c|c|c|}
\hline & $\begin{array}{c}\text { Superficie } \\
\left(\mathrm{km}^{2}\right)\end{array}$ & $\begin{array}{c}\text { Population } \\
\text { mi-2006 }\end{array}$ & $\begin{array}{c}\text { Densité } \\
\left(\mathrm{hab} / \mathrm{km}^{2}\right)\end{array}$ \\
\hline Argentine & 2780400 & 39000000 & 14 \\
\hline Bolivie & 1098580 & 9100000 & 8 \\
\hline Chili & 736480 & 16400000 & 22 \\
\hline Colombie & 1128700 & 46800000 & 41 \\
\hline Équateur & 272050 & 13300000 & 49 \\
\hline Pérou & 1279990 & 28400000 & 22 \\
\hline Venezuela & 912050 & 27000000 & 30 \\
\hline Total & 8208250 & 180000000 & 22 \\
\hline Amérique du Sud & 17867251 & 378000000 & 21 \\
\hline
\end{tabular}

$\mathrm{NB}$ : les marges andines de l'Argentine couvrent moins de 2 millions de $\mathrm{km}^{2}$ et rassemblaient environ 12 millions d'habitants en 2001.

Par son étirement sur $65^{\circ}$ de latitude, l'espace andin se déploie dans plusieurs grandes zones climatiques. De l'équateur au $55^{\mathrm{e}}$ parallèle sud, se succèdent ainsi toute une palette de climats : tropicaux humides en façade pacifique, équatoriaux et subéquatoriaux en façade orientale (Andes tropicales humides), semi-arides 
à arides à l'ouest de la cordillère, tropicaux humides à sec à l'est (Andes tropicales sèches), tempérés méditerranéens puis océaniques en façade occidentale, continentaux secs en façade orientale (Andes australes tempérées) et enfin froids à subantarctiques au-delà du $45^{e}$ parallèle (Andes australes froides). La continuité de l'imposante barrière orographique induit en outre, à partir du sud de l'Équateur, une dissymétrie climatique entre ses versants externes et les deux piémonts correspondants, dissymétrie renforcée par le courant marin froid de Humboldt. Au caractère semi-désertique à désertique du versant et du piémont occidental, entre le sud de l'Équateur et le Chili central, s'oppose le caractère hyperhumide du versant et du piémont oriental, au couvert forestier dense, entre le sud de la Colombie et le nord de la Bolivie. Au sud de la chaîne, inversement, le versant occidental tempéré et humide du sud du Chili s'oppose au versant en position d'abri et au piémont semi-aride, entre le sud de la Bolivie et la Patagonie argentine.

Enfin, le gradient d'altitude, déployé sur 5 à $7 \mathrm{~km}$ entre les basses terres des piémonts et les hautes terres des cordillères est à l'origine, comme dans toutes les montagnes, de fortes variations des conditions bioclimatiques et d'un étagement classique des formations végétales et des formes de mise en valeur, modifié par ailleurs par des effets d'exposition et de situation relative des lieux au sein du massif. La répartition de la population et la densité de l'occupation humaine ne sont cependant pas simplement déterminées par les contraintes orographiques, climatiques ou altitudinales (alternance de milieux inhospitaliers et d'espaces favorables à l'installation humaine).

\section{«Pleins et vides» du peuplement}

La cordillère des Andes et ses marges immédiates se déploient comme un ensemble de peuplement entre deux milieux démographiquement vides ou quasi vides. À l'ouest, c'est l'immense espace maritime du Pacifique, ici océan quasi absolu: les civilisations polynésiennes sont en effet situées à $6000 \mathrm{~km}$ des côtes sudaméricaines et l'archipel de Colón (les îles Galápagos, $8000 \mathrm{~km}^{2}$ et 20000 habitants) ou l'île de Pâques (3500 habitants) sont éloignés des côtes équatoriennes $(1000 \mathrm{~km})$ et chiliennes $(3600 \mathrm{~km})$. À l'est, s'étend la plus grande forêt tropicale de la planète drainée par le plus grand système hydrographique du monde: immense espace faiblement peuplé où les concentrations humaines s'égrènent le long des fleuves et des axes de communication terrestres.

La carte des densités de population aux niveaux national et infranational (premier niveau hiérarchique administratif) montre les "vides» et les "pleins» du peuplement (fig. 3). Elle coïncide en partie avec celle de la répartition des milieux : déserts côtiers ou d'altitude, Patagonie, cuvette amazonienne, Choco colombien, pour les "vides"; littoral tropical caraïbe, Andes septentrionales et centrales (où les foyers démographiques sont fort anciens), oasis de la côte pacifique, pour les "pleins", où se trouvent aussi les grandes concentrations urbaines millionnaires.

Comme l'ensemble de l'Amérique latine, les pays andins, ont eu en effet un développement urbain précoce ; ils ont un taux d'urbanisation élevé et connaissent une 
FIGURE 3 : ESPACE ANDIN ET MONDIALISATION : MÉTROPOLES, RESSOURCES, DISPARITÉS

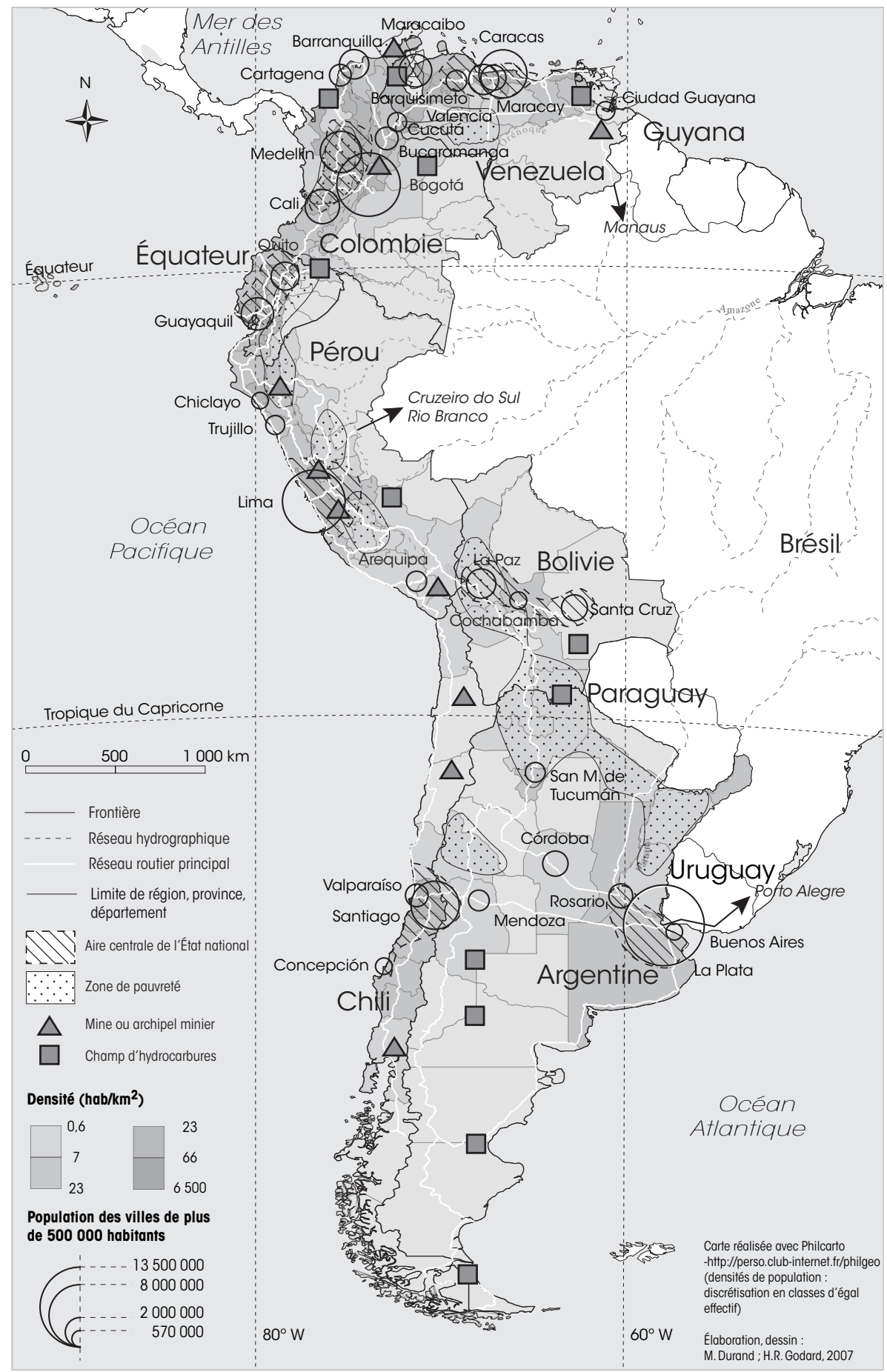

Sources: GeoHywe, 2007 (villes) ; organisations internationales ; recensements nationaux (pauvrełé) 
forte métropolisation. Près de 145 millions d'habitants, soit $80 \%$ de leur population, vit dans les villes et le taux d'urbanisation s'y échelonne entre $60 \%$ (Équateur et Bolivie) et près de $90 \%$ (Venezuela, Chili et Argentine). Les dix-neuf aires urbaines millionnaires regroupent $45 \%$ de leur population urbaine. Enfin, cinq métropoles approchant ou dépassant 5 millions d'habitants (Buenos Aires, Lima, Bogotá, Caracas et Santiago) totalisent 40 millions habitants, avec respectivement $39 \%, 39 \%, 24 \%, 20 \%$ et $34 \%$ de la population urbaine nationale. À l'exception de la Colombie et, dans une moindre mesure du Venezuela et de l'Équateur (Quito et Guayaquil), où le système urbain est hiérarchiquement mieux équilibré que celui des autres pays andins, on observe une concentration élevée de la population urbaine nationale (environ $40 \%$ à Buenos Aires et Lima) et de la population totale du pays (entre $29 \%$ et $35 \%$ ) dans l'agglomération-capitale ${ }^{2}$.

\section{Héritages partagés d'une histoire commune}

Plus encore que les fortes contraintes naturelles, la structuration territoriale de l'espace andin est aussi marquée par les héritages laissés, depuis l'époque précolombienne jusqu'à la mise en place d'États-nations, par une insertion précoce dans un mouvement de mondialisation dont on peut considérer que la découverte et la conquête de l'Amérique constitue une première étape.

Commandé de Cuzco (à 3400 m d'altitude) au cour des Andes, l'un des deux grands empires précolombiens de l'époque, peuplé d'une douzaine de millions d'habitants, occupait un territoire de près d'un million de $\mathrm{km}^{2}$. Réuni par les souverains incas en deux à trois générations, ses marges - amazoniennes notamment - échappaient en partie au pouvoir central. La conquête espagnole allait en bouleverser profondément les structures politiques, économiques et sociales, tout en élargissant l'assise territoriale. Trois siècles plus tard, la fragmentation de l'empire colonial espagnol donnait naissance, dans cette partie de l'Amérique, à sept États andins aux marges longtemps mal contrôlées.

\section{D'un empire à l'autre}

Les Espagnols avaient donc conquis, au Xvi ${ }^{e}$ siècle, l'empire du Tahuantisuyu, dont l'espace, maîtrisé de façon discontinue, s'étendait du nord de l'Équateur au Chili central et à la bordure orientale de l'Argentine. Organisé par les Incas, le coeur de l'Empire battait sur les hautes terres des Andes (cordillère et altiplano) du Pérou central jusqu'au lac Titicaca. Aire culturelle brillante qui avait vu se développer des structures politiques et socioéconomiques complexes, des systèmes agricoles et hydrauliques perfectionnés, des centres urbains puissants, l'empire avait imprimé sa marque sur l'évolution des populations andines et sur les formes d'utilisation de l'espace, dont les traces sont perceptibles jusqu'à nos jours. Ce système impérial autonome, qui n'avait que des relations ténues avec certains des autres groupes culturels

2 - Les données démographiques et urbaines sont tirées des dernières éditions des annuaires des organisations internationales. 
précolombiens dispersés en Amérique du Sud, s'effondra en quelques années sous le double effet de fragilités et dissensions internes et du choc de la conquête.

Malgré la diminution catastrophique de la population préhispanique aux $\mathrm{XvI}^{\mathrm{e}}$ et $\mathrm{XVII}^{e}$ siècles et le métissage généralisé issu des apports successifs européens, africains et asiatiques, les minorités "indiennes» restent encore très présentes. Nombreuses dans les Andes centrales, cour de l'Empire inca aujourd'hui en position de périphérie géographique et économique, leur part dans la population totale décroît vers le nord et vers le sud - à l'exception des noyaux de peuplement préhispanique Mapuche et Araucan du Chili ${ }^{3}$. Populations décimées et trop longtemps exclues, ces vaincus de l'histoire reviennent depuis quelques années sur le devant de la scène andine et parfois au plus haut niveau (élection de Evo Morales, président bolivien depuis 2006). Le contrôle colonial des territoires conquis s'appuya largement sur un réseau de villes : villes préhispaniques soumises (juxtaposition ou superposition de site, après une éventuelle destruction de la cité existante) et nouvelles fondations destinées à assurer la sécurité des territoires, les relations avec la métropole, l'acheminement des esclaves, le contrôle et l'exploitation des populations, des ressources et des espaces. Les créations urbaines furent nombreuses, tant sur les littoraux caraïbe et pacifique que sur les hautes terres, avec une logique de maillage urbain qui associait les anciennes et les nouvelles capitales, les villes de l'intérieur et les ports ${ }^{4}$.

Dans un contexte que l'on peut qualifier de première mondialisation, la colonisation espagnole - la plus longue mise en place par les puissances européennes - dura trois siècles. Elle s'appuyait sur une organisation territoriale dont le maillage évolua au fil du temps. La vice-royauté du Pérou, dont Lima était la capitale, engloba d'abord l'ensemble des possessions espagnoles d'Amérique du Sud jusqu'à l'actuel Panama inclus, avec une subdivision en unités de rang inférieur, les audiences (Quito, Charcas...) ou les capitaineries générales (Chili et Venezuela); elle fut divisée au $\mathrm{XVIII}^{\mathrm{e}}$ siècle, avec la création des vice-royautés de NouvelleGrenade (Bogotá) et du Río de La Plata (Buenos Aires).

\section{Les États-nations et la consolidation de leur souveraineté territoriale}

Au début du $\mathrm{XIX}^{\mathrm{e}}$ siècle, les élites créoles urbaines, opposées à l'administration coloniale, furent à l'origine du mouvement d'indépendance précoce de cet empire espagnol. Partis des marges peu peuplées de l'empire, Simón Bolívar déclara l'indépendance du Venezuela en 1821 (tentative avortée de F. de Miranda en 1811)

3 - Dans les années 1990, la part des populations de souche amérindienne dans la population totale est estimée supérieure à $50 \%$ en Bolivie, proche de $50 \%$ au Pérou, de l'ordre de $35 \%$ en Équateur et de $10 \%$ au Chili. Dans les autres pays andins, elle serait inférieure à $2 \%$ (voir le chapitre de Virginie Baby-Collin in S. Velut (dir.), L'Amérique latine, Paris, SEDES-CNED, 2006 et, sur l'ambiguïté des critères de définition d'appartenance ethnique ou linguistique, le chapitre de Vincent Gouëset in F. Dureau, V. Gouëset et É. Mesclier (dir.), Géographies de l'Amérique latine, Rennes, Presses universitaires de Rennes, 2006).

4 - Les capitales de la Bolivie, de l'Équateur et de la Colombie, situées à plus de 2500 m d'altitude, sont les plus hautes du monde. 
et José de San Martín proclama celle de l'Argentine en $1816^{5}$; la rencontre des deux libertadores eut lieu à Guayaquil en 1822. En dépit du rêve unificateur bolivarien - les tentatives d'union ayant échoué : Grande Colombie réunissant les Andes du Nord de Caracas à Guayaquil (1819-1830) et éphémère confédération péruano-bolivienne l'empire fut fragmenté pour donner naissance, entre 1810 et 1830, aux États-nations andins actuels ${ }^{6}$. En revanche, le système économique et social issu de la conquête n'a pas fondamentalement changé après les indépendances : le modèle d'extraversion économique en vigueur s'est maintenu et les processus ségrégatifs se sont accentués au sein de ces sociétés historiquement inégalitaires, segmentées et hiérarchisées.

Si la définition des frontières a donné lieu à de nombreux conflits bilatéraux limités aux $\mathrm{XIX}^{\mathrm{e}}$ et $\mathrm{XX}^{e}$ siècles - certains tracés sont encore en discussion -, les guerres internationales, motivées par les visées expansionnistes de certains États (Brésil, Argentine, Chili) pour s'approprier des territoires ou des matières premières, ont été peu nombreuses. La Bolivie a été le pays le plus affecté par les atteintes à une intégrité territoriale historiquement revendiquée. Au cours de la guerre du Pacifique ou guerre du salpêtre (1879-1883), le Chili envahit la Bolivie alliée au Pérou. Le Pérou y perdit la région d'Arica et la Bolivie fut privée de son département littoral (environ $125000 \mathrm{~km}^{2}$ autour du port d'Antofagasta) et donc d'un accès à la mer; l'Argentine profita de la guerre du Pacifique pour avancer en Patagonie au détriment des Chiliens occupés sur un autre front. Au cours de la guerre qui l'opposa au Brésil en 1903-1904, la Bolivie perdit ensuite le territoire de l'Acre (180000 km²). Enfin, lors de la guerre du Chaco entre Bolivie et Paraguay (1932-1935), pour cette plaine orientale censée receler des ressources pétrolières, la Bolivie fut encore amputée de près de $225000 \mathrm{~km}^{2}$. En un demi-siècle, la superficie du territoire bolivien se trouvait ainsi réduite d'un tiers ${ }^{7}$, avec des conséquences spatiales (enclavement), un ressentiment de la population bolivienne toujours d'actualité et des relations qui restent difficiles avec le Chili.

Hormis quelques grands conflits parfois meurtriers, les différends frontaliers pour le contrôle de vastes territoires quasi vides de population en Amazonie ou dans les Andes australes ont été résolus pacifiquement ou au prix d'escarmouches, de menaces de guerre ou de guerres de portée très limitée. Les rectifications de frontières, le plus souvent, ont été conduites par des arbitrages internationaux. La dernière en date, à la suite du conflit frontalier de la cordillère du Condor, a fait l'objet d'un accord de paix entre l'Équateur et le Pérou en 1995, après une série de

5 - Autour de 1800, l'Amérique andine rassemblait environ 5 millions d'habitants, bien moins qu'avant la conquête; vers 1850, elle en abritait moins de 11 millions; en 1900, elle en regroupait 21 millions.

6 - Cependant, les frontières des États-nations ne se superposent pas aux anciennes limites coloniales. Seulement $27 \%$ des frontières terrestres actuelles sont d'origine coloniale; au début des années 1990, 26 \% étaient issues de guerres, 34 \% avaient été négociées ou imposées et 13 \% étaient arbitrées ou en litige (voir M. Foucher, Fronts et frontières..., Paris, Fayard, 1991, annexe p. 9).

7 - Entre 1865 et 1870, la guerre de la Triple alliance a vu s'affronter le Paraguay et la coalition composée du Brésil, de l'Argentine et de l'Uruguay. Le Paraguay a perdu la moitié de sa population et $140000 \mathrm{~km}^{2}$ au profit des vainqueurs. Enfin, dans les années 1910, les frontières brésiliennes ont été rectifiées aux dépens de la Colombie et du Venezuela. 
conflits limités survenus entre les deux pays, tout au long des $\mathrm{XIX}^{e}$ et $\mathrm{XX}^{e}$ siècles, pour le contrôle des terres amazoniennes. Au total, et bien que des litiges subsistent, les frontières terrestres sont stabilisées; en revanche, des négociations se poursuivent pour définir l'extension des zones maritimes économiques exclusives ou régler les différends portant sur l'Antarctique.

\section{Des périphéries nationales mal contrôlées}

En dépit des marques de souveraineté et de légitimité des États (installation de bornes, création de parcs nationaux), les périphéries géographiques difficiles d'accès et peu peuplées, comme les versants andins orientaux et leurs piémonts amazoniens, se caractérisent par leur perméabilité au développement d'activités illégales : flux incontrôlés de personnes et refuges de guérillas, trafics de marchandises et productions illicites, notamment la transformation et l'acheminement de la cocaïne pour le marché local et international, la présence de groupes armés et l'économie de la drogue étant souvent liées.

Producteurs quasi exclusifs de feuilles de coca et de cocaïne dans le monde, la Colombie, le Pérou et la Bolivie fournissent les marchés états-unien et européen à partir de la Colombie, leader mondial de la transformation de la feuille de coca et de la production de pâte base, de la vente en gros et de la distribution de cocaïne. En 2005, la Colombie cultivait plus de la moitié des surfaces plantées en cocaïers dans le monde ( 86000 ha, principalement concentrés en Amazonie dans les régions de Putumayo-Caquetá et de Meta-Guaviare) et disposait de $70 \%$ de la production potentielle de feuilles de coca (plus de 170000 tonnes) et de la fabrication de cocaïne (640 tonnes) ${ }^{8}$. Depuis la fin des années 1990, marquée par la lutte accrue contre la culture et la production, largement financée et contrôlée par les États-Unis, le partage des tâches entre les trois pays s'est intensifié : réduction des superficies cultivées en Colombie mais accroissement au Pérou et en Bolivie depuis 2000; fabrication de la cocaïne en Colombie alors que les deux autres pays n'élaborent que des produits intermédiaires (pâte de coca et cocaïne base). Dans l'ensemble de la région, les résultats des politiques d'éradication sont mitigés : malgré la diminution des superficies cultivées (- $28 \%$ depuis 2000), le potentiel de fabrication de cocaïne reste stable (910 tonnes en 2005 contre 880 en 2000) en raison de l'accroissement des rendements de la feuille de coca. Si les Colombiens maîtrisent l'ensemble de la filière (culture et production, transformation et acheminement du produit final jusqu'au Mexique qui assure le passage de la cocaïne aux États-Unis, dernière étape du trafic), on assiste depuis quelques années à une recomposition des réseaux, avec la montée en puissance des cartels mexicains qui réussissent, notamment au Pérou, une percée leur permettant d'organiser les exportations et de s'affranchir des intermédiaires colombiens.

Dans ces marges économiques et territoriales où s'articulent le monde et l'antimonde, deux facettes d'un même système, ces formes particulières de mise en

8 - UNODOC, volumes 1 et 2. Il semble qu'il s'agisse des données disponibles les plus fiables. 
valeur de l'espace et les revenus qu'elles assurent pèsent sur le PIB des États et accroissent les inégalités intranationales.

\section{Les disparités spatiales, économiques et sociales}

Au sein des pays du Sud, l'Amérique latine se caractérise par les niveaux de développement humain les plus élevés, les pays andins se situant dans la moyenne du souscontinent. Cependant, ces performances ne doivent pas masquer la situation préoccupante dans laquelle se trouvent plongés nombre de pays latino-américains car, au-delà de la valeur élevée des indicateurs de base, les contrastes socioéconomiques et spatiaux sont marqués au niveau des ensembles régionaux comme au niveau infranational. Les conditions sociales se dégradent - bien que la croissance du PIB soit significative depuis le début du $\mathrm{xxI}^{\mathrm{e}}$ siècle, du moins dans les pays andins - et les inégalités se creusent.

\section{Des indicateurs de développement assez élevés, des inégalités marquées}

En 2004, l'Indicateur de développement humain (IDH) de l'Amérique latine $(0,770)$ était supérieur à l'IDH mondial $(0,741)$ et il dépassait largement celui de l'ensemble des pays en développement $^{9}$. L'IDH des sous-ensembles régionaux était proche de la moyenne latino-américaine : 0,784 pour l'Amérique du Sud et 0,789 pour l'Amérique andine. L'analyse des autres marqueurs démographiques et socioéconomiques confirme la situation relativement "privilégiée» de l'Amérique latine en général et de certains pays en particulier, dont les indicateurs se rapprochent de ceux des pays industrialisés (notamment en termes de croissance démographique et de niveau éducatif et sanitaire).

Mais la moyenne andine cache de fortes disparités entre pays (fig. 4 et 5). L'IDH s'échelonne entre 0,863 (Argentine, $36^{\mathrm{e}}$ rang mondial, devant de nombreux pays $\mathrm{d}^{\prime}$ Europe orientale) et 0,692 (Bolivie, $115^{\mathrm{e}}$ rang). Les contrastes se lisent aussi dans l'analyse des situations démographiques : le taux de natalité est inférieur à $20 \%$ dans les pays du Cône sud et supérieur à $25 \%$ dans ceux des Andes centrales (31\%o en Bolivie); la part des moins de 15 ans dans la population totale oscille de $25 \%$ à $39 \%$; le taux de mortalité infantile varie dans un rapport de 1 à 7 . Si le taux d'urbanisation des sous-ensembles sud-américain et andin, proche de $80 \%$, est le plus élevé de l'Amérique latine, il recouvre de fortes disparités. L'hétérogénéité des niveaux de vie est également très marquée: le PIB annuel par habitant varie de 1 à 4 (Bolivie : moins de 3000 dollars, Argentine : plus de 13000 dollars).

La situation des pays andins, comme celle de l'Amérique latine en général, semble paradoxale. Les indicateurs, assez élevés, ont beaucoup progressé depuis le milieu des années 1970. L'IDH moyen des pays andins a augmenté de plus de 18 \% entre 1975

9 - Les données statistiques présentées dans cette partie sont issues des publications annuelles des organisations internationales (CEPAL, UNFA, PRB et UNDP). 


\section{FiguRE 4 : DISPARITÉS SOCIOÉCONOMIQUES ET EXCLUSION DANS LES PAYS ANDINS}

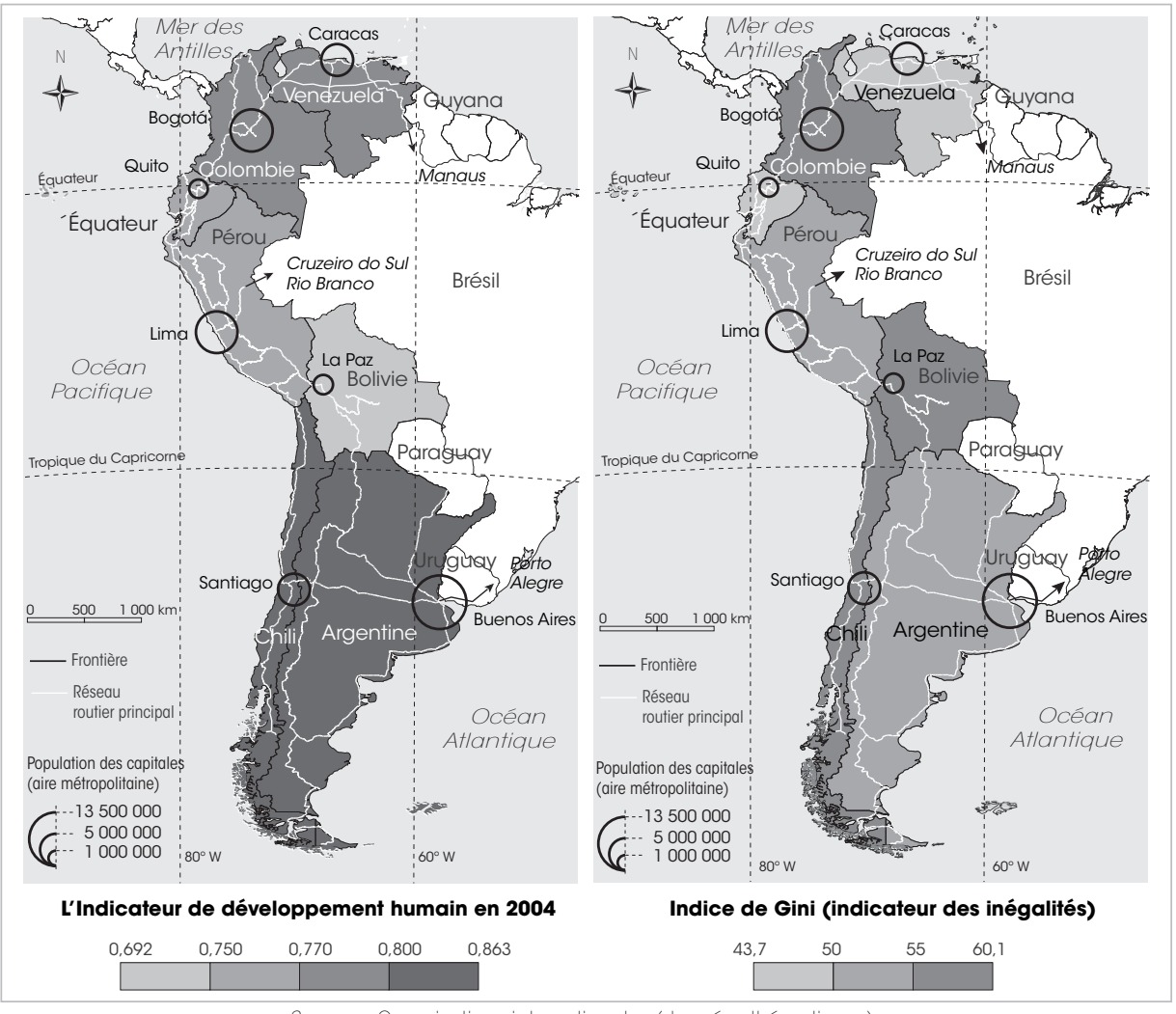

Sources : Organisations internationales (données thématiques)

et 2004 : l'amélioration a été supérieure à la moyenne au Chili, en Colombie et en Bolivie, où la valeur était à peine supérieure à 0,5 en 1975 (+35\%); en revanche, elle a été inférieure à $10 \%$ en Argentine, touchée par plusieurs crises économiques, et au Venezuela, qui a enregistré le plus faible taux de croissance de la région entre 1995 et 2004 (il semble que la manne pétrolière n'ait pas eu les retombées sociales espérées). Cependant, malgré des progrès évidents, la répartition de la richesse nationale reste très inégalitaire et les disparités régionales sont fortes. Ainsi, selon les données de la Commission économique pour l'Amérique latine et les Caraïbes (CEPALC), 13 millions de Latino-Américains seraient sortis de la pauvreté entre 2003 et 2005 : cependant, plus de 200 millions d'habitants restent pauvres (40\% de la population) et près de 90 millions d'entre eux (17 \%) vivraient encore dans l'indigence. Si au début des années 2000, 23 \% de la population des pays andins vivait avec moins de 2 dollars par jour - le taux national variant dans un rapport de 1 à 4 (10 \% au Chili contre $42 \%$ en Bolivie) -, le rapport entre la frange de la population la plus aisée et celle qui vit dans la pauvreté est très élevé (fig. 4). L'Amérique latine est l'aire géographique la plus inégalitaire au monde; l'indice de Gini, qui permet de mesurer les inégalités ( 0 correspond à l'égalité parfaite et 100 à l'inégalité "parfaite»), y est supérieur à 50 dans onze pays et partout supérieur à 40, la Bolivie détenant le triste record du continent $(60,1)$, dépassé seulement par sept pays d'Afrique centrale et australe. Le Chili est le seul pays 
latino-américain à avoir atteint l'un des objectifs du millénaire en 2004 : réduire de moitié le nombre d'habitants vivant avec moins d'un dollar par jour.

Tous les indicateurs, démographiques, sociaux ou économiques révèlent donc des disparités marquées entre les pays andins, fractures qui se manifestent aussi à l'échelon infranational et recoupent les oppositions ethnolinguistiques, hommes / femmes, ville/campagne, régions dynamiques / régions en crise.

\section{FIGURE 5 : DisPARITÉS DÉMOGRAPHIQUES ET URBANISATION DANS LES PAYS ANDINS}

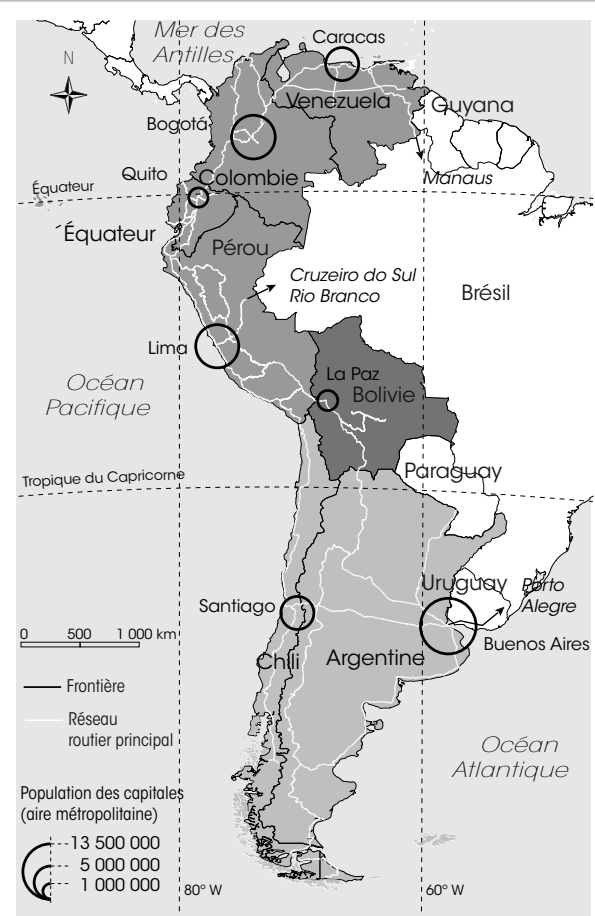

Les moins de 15 ans en 2004

39

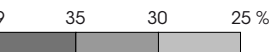

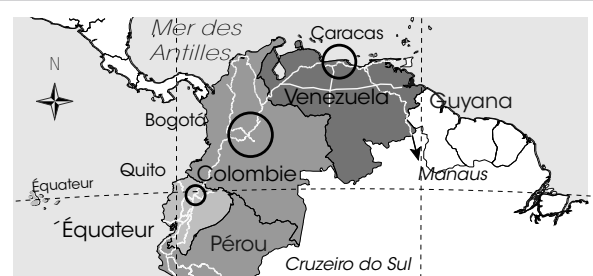

Brésil

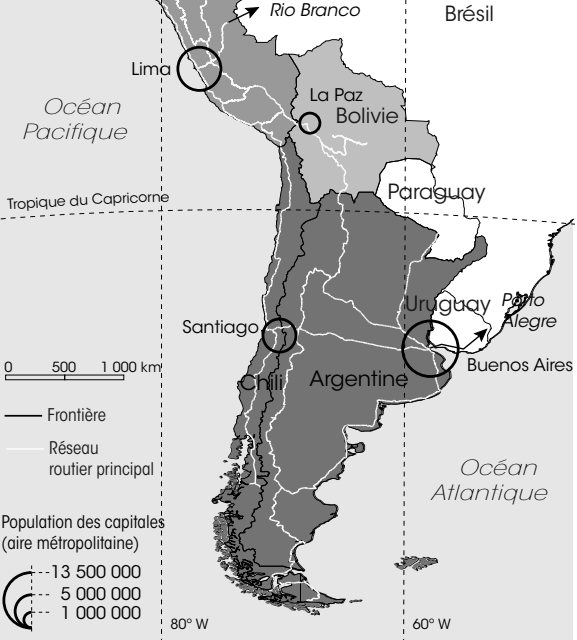

Le taux d'urbanisation au début du $X X I^{e}$ siècle

93

$80 \quad 70 \quad 62 \%$

Sources : Organisations internationales (données thématiques).

\section{Sept pays, quatre groupes}

La lecture des cartes précédentes donne à voir une hiérarchie démographique et socioéconomique des pays andins quasi identique, quel que soit l'indicateur considéré. La figure 6 est une synthèse, construite à partir d'une vingtaine de variables non redondantes, qui permet de définir une typologie. Les dix-neuf variables retenues prennent en compte les caractéristiques démographiques (taux d'accroissement naturel, taux de mortalité infantile, espérance de vie...), les conditions d'éducation et de santé (malnutrition, alphabétisation...), l'inégale répartition des richesses et le degré de pauvreté et d'exclusion (PIB par habitant, part de la population vivant au-dessous de la ligne de pauvreté...), le niveau de confort (accès à 
FiguRE 6 : TYPOLOGIE DES PAYS DE L'AMÉRIQUE ANDINE À PARTIR DE 19 INDICATEURS



l'eau potable, qualité de l'assainissement, nombre d'internautes...) et les caractéristiques économiques (part de la production industrielle dans la composition du PIB, structure des exportations et montant de l'aide publique au développement par habitant). Les pays ont ensuite été classés par ordre décroissant, variable par variable: un point a été attribué au pays qui obtenait le meilleur score et sept points à celui qui terminait dernier (le total théorique possible est donc compris entre 19 et 124 points). La carte, qui rend compte du total de points obtenus par chacun des pays, permet d'ébaucher une typologie en quatre classes.

L'Argentine et le Chili, avec respectivement 33 et 36 points, obtiennent les scores les plus faibles. Aux marges méridionales de l'ancien empire espagnol, ces pays «neufs» du Cône sud, dont l'importante population d'origine européenne est issue des migrations des $\mathrm{XIX}^{\mathrm{e}}$ et $\mathrm{XX}^{\mathrm{e}}$ siècles, disposent de ressources naturelles considérables (Chili), d'une agriculture et d'un élevage tournés vers l'exportation et d'une base industrielle relativement solide (Argentine).

Suivent ensuite, loin derrière les pays du Cône sud, le Venezuela et la Colombie, 74 points chacun. Terres très métissées, à la rencontre des mondes andin et caraïbe, le Venezuela tire l'essentiel de ses revenus des ressources pétrolières tandis que la Colombie, avec une base agricole et industrielle plus solide, dispose d'une économie plus diversifiée, moins sujette aux aléas des cours internationaux.

Le troisième groupe rassemble l'Équateur et le Pérou, avec respectivement 89 et 96 points. Jadis au cœur des anciens empires inca et espagnol, ces pays où la population d'origine amérindienne reste supérieure au tiers de la population totale disposent d'importantes ressources naturelles, d'une agriculture à deux visages - petites exploitations paysannes traditionnelles; grandes et moyennes entreprises agro-exportatrices (Équateur notamment) - et d'une industrie qui s'étoffe.

Enfin, la Bolivie ferme la série avec 133 points. Ce pays enclavé, où la majorité de la population d'origine amérindienne est en situation de marginalisation et d'exclusion extrêmes, est en proie à un régionalisme exacerbé. L'agriculture y est aussi contrastée qu'au Pérou voisin et le secteur industriel est faible. C'est le seul pays d'Amérique du 
Sud dont le niveau de développement économique et humain est proche de celui de la plupart des pays de l'isthme, le sous-ensemble latino-américain le plus défavorisé.

Une analyse à des niveaux plus fins ferait ressortir les différenciations infranationales laissant apparaître des disparités régionales qui soulignent de forts contrastes structurels. Ainsi l'altiplano bolivien rural et pauvre, habité par une population de souche amérindienne, s'oppose à l'Oriente, plus urbanisé et industrialisé, richement doté en gisements de gaz naturel et aux puissantes élites créoles.

Si la vision réductrice d'un sous-ensemble andin (ou latino-américain) homogène doit donc être nuancée, il n'en demeure pas moins qu'au-delà des disparités - et des clivages régionaux propres à des États dont l'étendue varie de un à dix - on constate une parenté certaine dans les dynamiques territoriales et les formes d'organisation spatiale, en lien avec des tendances lourdes, économiques, sociales et politiques, qui transcendent souvent les frontières nationales.

\section{Des structures spatiales récurrentes}

Exposés au vent de l'internationalisation des échanges et de la mondialisation, les pays andins et leurs sociétés profitent parfois de la dérégulation des marchés et des orientations économiques néo-libérales comme ils en subissent souvent les conséquences. Les centres de commandement urbain et les aires centrales, les régions géographiques en périphérie des États - y compris les zones de très faible peuplement humain où se trouvent nombre de gisements miniers et d'hydrocarbures intensément exploités - connaissent donc des évolutions qui sont indissociables des dynamiques du système monde et de ses marchés (fig. 3).

\section{Des aires centrales nationales et des métropoles inégalement connectées au système monde}

La forme d'exploitation des ressources des territoires (pour les besoins de la métropole espagnole dès la conquête, puis pour ceux des pays industrialisés à partir de la formation des États-nations) comme l'asymétrie des échanges n'ont pas foncièrement changé. Des révolutions politiques, sociales, économiques et technologiques ont bouleversé l' «Ancien» et le "Nouveau» Mondes et des modèles de développement se sont succédé, mais l'inégalité des relations entre les pays andins et le reste du monde demeure. Au $\mathrm{xxI}^{\mathrm{e}}$ siècle, la structuration des territoires nationaux, leur organisation spatiale et l'accentuation des disparités régionales portent la marque de cette complémentarité déséquilibrée entre centres de commandement mondiaux et espaces sous influence dont fait partie l'aire andine.

Dans chacun des États, une aire centrale - «noyau dur» d'espaces densément occupés, intégré dans les réseaux mondiaux, à partir et autour duquel s'est construit l'espace national - rassemble une ou plusieurs métropoles qui contrôlent des régions d'étendue variable et drainent les productions destinées au marché mondial (minerais, hydrocarbures, produits agropastoraux). L'extension et le poids dans l'économie nationale, l'influence mondiale de chaque aire centrale 
dépendent, entre autres, de la taille du pays et de l'importance de sa population. Chacune relève également de legs historiques - importance des foyers de population amérindienne, de l'apport des vagues migratoires successives postérieures aux indépendances, de l'intensité du processus d'équipement et de modernisation du territoire national, du degré de métropolisation -, des ressources exploitables, mais aussi de certaines contraintes orographiques ou climatiques.

Trois types d'aires centrales peuvent être identifiés. Le premier groupe rassemble les pays dont la capitale concentre la quasi-totalité des fonctions de commandement. En Argentine, Buenos Aires, première agglomération latino-américaine dans les années 1920 (1,8 million d'habitants) et troisième en 2007 derrière Mexico et São Paulo, à la fois capitale et port, domine sans partage l'espace national ${ }^{10}$. En bordure centre-orientale de la pampa, elle draine les productions agropastorales d'une région plus grande que la France. La mégapole porteña, porte d'entrée des migrants venus d'Europe, port principal d'exportation et cœur économique du pays, a fait obstacle à un développement plus équilibré du réseau urbain national. En effet, malgré la superficie importante du pays, les autres grands centres urbains n'ont qu'une influence régionale. Le Pérou et le Chili présentent un cas de figure analogue : leur capitale administrative coloniale ne se trouvant pas directement sur l'océan sans en être très éloignée, les Espagnols les avaient dotés d'un débouché vital vers la métropole. Aujourd'hui, le port de Callao fait partie de l'agglomération de Lima, qui concentre pouvoir et richesses, et celui de Valparaíso, à une centaine de kilomètres de Santiago, est sous la dépendance directe de la métropole chilienne. Le second type correspond aux pays dont l'aire centrale s'organise autour de plusieurs métropoles. En Équateur, l'aire centrale s'étend de Quito à Guayaquil, située à $400 \mathrm{~km}$ de la capitale : ces deux agglomérations, aux rôles et aux économies complémentaires, se partagent les fonctions de commandement. Dans la Bolivie privée d'accès à la mer, l'espace central des hautes terres andines, entre le lac Titicaca et les vallées de Cochabamba, s'est d'abord structuré autour de La Paz, ville principale qui bénéficiait de la rente minière et siège du gouvernement (Sucre étant la capitale constitutionnelle); avec les crises qui secouent les hautes terres depuis les années 1980, la montée en puissance, depuis les années 1960, de Santa Cruz, la métropole des terres basses du piémont oriental, a introduit une dyarchie majeure. En situation intermédiaire entre le deuxième et le troisième groupe, le Venezuela se singularise par le développement d'une aire centrale littorale, sur près de $300 \mathrm{~km}$, où un chapelet de centres urbains est dominé par la métropole de Caracas qui inclut le port de La Guaira. Quelques pôles périphériques (Maracaïbo, Barquisimeto) émergent mais restent cependant sous l'influence de Caracas. La Colombie enfin se caractérise par un réseau urbain polycentrique mieux équilibré et davantage hiérarchisé. Si le "triangle d'or ${ }^{11}$ » Bogotá-Medellín-Cali structure le cœur de l'aire centrale colombienne, d'autres pôles jouent un rôle régional important; et plusieurs métropoles disposent de fonctions qui dépassent les limites de

10 - Le port de Buenos Aires n'a été construit qu'au XIX ${ }^{e}$ siècle. Pendant la période coloniale, la fonction portuaire n'a pas été développée en raison du monopole du port de Callao jusqu'en 1776.

11 - Voir le chapitre de Jean-Paul Deler «Improbable Colombie», in C. Bataillon, J.-P. Deler et H. Théry, Amérique latine, 1991, réédition Paris, Belin, Géographie universelle, 2000. 
leur région : ports de Barranquilla et Cartagena de Indias [Carthagène], pôle industriel de Medellín, centre touristique de Carthagène.

On notera que plusieurs des aires métropolitaines de l'Amérique andine appartiennent en outre au cercle restreint des cités géantes (à la fois mégavilles et villes mondiales) qui constituent l'Archipel mégalopolitain mondial (AMM) ${ }^{12}$, ce réseau des centres de contrôle et de commandement des dynamiques planétaires. Dans la classification établie, en 1999, par le Groupe d'études sur la globalisation et les villes mondiales $(\mathrm{GaWC})^{13}$ et qui concerne cinquante-cinq métropoles élevées au rang de ville mondiale, figurent trois mégapoles andines: Buenos Aires, Caracas et Santiago. Elles n'y apparaissent cependant qu'au troisième et dernier rang hiérarchique. Mais les auteurs identifient aussi deux autres pôles, Bogotá et Lima, qui pourraient, à terme, entrer dans cette catégorie. Distantes les unes des autres de plusieurs milliers de kilomètres mais interconnectées, les grandes villes de cet archipel métropolitain andin, qui captent les flux majeurs de la mondialisation, ont bien souvent des relations plus intenses entre elles qu'avec une grande partie de leur environnement immédiat : le «reste» de l'espace national constituant une sorte de périphérie qui n'est toutefois pas homogène.

\section{Des régions et des enclaves pleinement intégrées aux marchés mondiaux}

Grandes régions de production agropastorale souvent partie intégrante de l'aire centrale du pays, ou enclave minière, touristique ou militaire en situation «insulaire» au sein du reste de l'espace national mais intégrée au système monde, offrent des modes d'organisation spatiale caractéristiques. Trois éléments déterminent le système spatial correspondant: un point d'intérêt (gisement minier, complexe touristique intégré, base militaire) ou une aire de ressources (région agricole relativement homogène ou vaste espace de colonisation d'exploitation discontinue); un axe principal (ferroviaire ou fluvial, routier, voire couloir aérien) ou un réseau hiérarchisé de voies secondaires branchées sur un axe principal qui relie la zone de production au débouché vers l'extérieur; un port d'exportation, parfois spécialisé, ou un aéroport international, points d'entrée et de sortie des flux de passagers du tourisme national, continental, ou des pays de la Triade (Amérique du Nord, Union

12 - Voir O. Dollfus, La mondialisation, Paris, Presses de Sciences Po, 1997. et P. Veltz, Mondialisation villes et territoires, Paris, Presses universitaires de France, 1997.

13 - Au début des années 1990, plusieurs chercheurs ont proposé différents systèmes de classification des villes mondiales. Le GaWC (département de géographie de l'université de Loughborough au Royaume-Uni) a mis au point une méthode, fondée sur une sélection de firmes opérant dans le secteur des services supérieurs, permettant de hiérarchiser les grandes villes du monde. Parmi les 122 villes retenues pour leur place à l'échelle mondiale dans les domaines de la comptabilité, la publicité, la finance et les services juridiques, 55 ont été qualifiées de villes mondiales : 10 appartiennent au niveau hiérarchique supérieur alpha, 10 au niveau intermédiaire bêta (dont São Paulo et Mexico) et 35 au niveau inférieur gamma (Voir J. V. Beaverstock, R. G. Smith et P. J. Taylor, «A Roster of World Cities», Cities, 16(6), 1999, p.445-458, accessible en ligne : http://www.lboro.ac.uk/gawc/ $\mathrm{rb} / \mathrm{rb} 5 . h \mathrm{tml})$. Toute classification est bien sûr discutable, mais celle-ci a le mérite de la précision et de la clarté. S'il est probable que la classification varierait en fonction des services supérieurs sélectionnés, elle ne serait pas fondamentalement différente. 
européenne et Asie du Nord-Est). Ce modèle de l'espace extraverti peut être altéré par certaines spécificités : ainsi les bases militaires contrôlées par les États-Unis, dont les missions sont essentiellement la maîtrise de l'espace aérien et maritime et l'intervention ponctuelle au sol, sont des espaces fermés qui regroupent toutes les infrastructures nécessaires et elles n'ont pas besoin d'être reliées au reste du territoire national qui les héberge (Colombie, Équateur); ou bien les hydrocarbures et le gaz destinés au marché sous-continental ou andin qui transitent par des réseaux de tubes dont le tracé ignore les limites des États où ils sont exploités.

L'extraversion économique et la logique exportatrice ont suscité, dans chaque pays, l'essentiel du développement des infrastructures, portuaires et ferroviaires à partir des années 1860, puis routières et aéroportuaires (liaisons intérieures et internationales) à partir des années 1950. Ces progrès techniques ont stimulé les transformations urbaines ainsi qu'un premier développement industriel des centres exportateurs et des pôles urbains principaux, grâce aux revenus des productions agropastorales et des matières premières exportées. Ils ont favorisé aussi, par le désenclavement de certaines régions, l'intégration nationale. L'extension du réseau ferré pour drainer les produits destinés à l'exportation a ainsi joué un rôle intégrateur en Argentine, au Chili et, dans une moindre mesure, au Pérou et en Bolivie. Dans d'autres pays comme la Colombie ou le Venezuela, les tronçons non reliés entre eux étaient construits sans perspective nationale, l'Équateur faisant exception avec l'ouverture d'un chemin de fer d'intérêt national qui ne fut pas conçu pour l'exploitation et l'exportation de matières premières.

Les grandes mines ou les gisements d'hydrocarbures représentent l'archétype de l'espace fermé - véritables enclaves au sein du territoire national, souvent contrôlées par des capitaux étrangers - dont le système spatial simple s'articule autour de deux points, les lieux de production et ceux d'exportation, reliés par une voie d'acheminement de la production. La zone de ressources, plus étendue, réunit de nombreux sites de production reliés à un axe principal par une arborescence de voies secondaires qui drainent minerais ou produits agropastoraux vers les lieux de conditionnement et/ou d'exportation; ainsi les archipels miniers polymétallifères des Andes du Sud (du Pérou et de la Bolivie au nord du Chili), les champs pétrolifères et les zones d'extraction de bois du bassin amazonien. Seules les grandes régions de colonisation agricole, comme les plaines du Guayas (cacao et banane), les Andes et leurs marges entre Antioquia et Cauca (café), la Pampa argentine (céréaliculture, élevage), ont permis un développement spatial auréolaire à partir d'un pôle urbain (Guayaquil, Medellín, Buenos Aires), qui a donné naissance à des réseaux urbains et de communication relativement hiérarchisés.

\section{Des espaces périphériques aux dynamiques multiples et aux niveaux hétérogènes d'intégration}

Aux marges de ces aires centrales ou des quelques grandes régions urbaines, mais également des régions les plus intégrées aux sollicitations des marchés mondiaux, le reste du territoire national constitue un ensemble spatial périphérique où les formes d'articulation et les niveaux d'intégration au processus de mondialisation 
restent très variables et où les dynamiques d'évolution sont extrêmement hétérogènes. Espace à la fois périphérique, au sein de chaque pays, et globalement en position «interstitielle», entre les grandes métropoles nationales ou les principales régions urbaines du sous-continent, c'est une sorte patchwork de territoires régionaux, quelquefois étroitement locaux, plus ou moins affectés par l'effet de "tunnel» lié au fonctionnement du modèle d'organisation de l'espace en «moyeux et rayons » (hubs and spokes). On esquisse ici une typologie de ces territoires périphériques, dans leur degré variable d'articulation aux dynamiques de la modernité.

Le réseau des villes moyennes en constitue le premier type, sous la forme d'une constellation de plus de quatre-vingts centres secondaires (entre 100000 et 500000 habitants), inégalement répartis dans les sept pays. Souvent assez bien reliés aux métropoles nationales (routes, aéroport), ces centres disposent, pour les plus importants, de services de niveau supérieur et parfois d'industries modernes; ils structurent en partie l'espace périphérique, à l'interface des sociétés rurale et urbaine, et dynamisent souvent l'économie dans leur environnement rural immédiat. Cumaná, Mérida, au Venezuela ; Montería, Neiva ou Pasto, en Colombie; Cuenca ou Manta, en Équateur ; Iquitos, Juliaca-Puno ou Piura, au Pérou; Sucre ou Tarija, en Bolivie; Talca et Temuco, au Chili; Bahía Blanca, Corrientes ou Neuquén, en Argentine, en sont des exemples.

Certains secteurs transfrontaliers, notamment au passage des grandes voies terrestres (la route panaméricaine essentiellement), forment un deuxième type d'espaces périphériques. Ici, les formes d'articulation et les dynamiques économiques se nourrissent de l'accroissement, encore modeste mais réel, des flux d'échanges entre les pays andins, des effets classiques du différentiel de pouvoir d'achat des monnaies (contrebande aussi florissante que l'informalité et la corruption) et, parfois, de la mise en œuvre de projets binationaux de développement local. Ces secteurs frontaliers incorporent les villes voisines les plus proches, stimulant les activités des doublets urbains : San Cristóbal et Cúcuta (Venezuela-Colombie), Ipiales et Tulcán (ColombieÉquateur), Machala et Tumbes (Équateur-Pérou), Tacna et Arica (Pérou-Chili). On peut considérer quelques zones franches portuaires comme une variante de ce type, mais à un niveau d'intégration supérieur (flux internationaux nord-sud et industries d'exportation) comme à Antofagasta et Arica (Chili) ou Santa Marta (Colombie).

D'autres territoires, qui disposent d'avantages comparatifs pour certains créneaux de productions ou prestations destinées au marché international (et/ou national), constituent un troisième type. Ce sont souvent de petites zones rurales où, du fait de conditions environnementales spécifiques, se maintient ou se développe une production locale agropastorale de qualité, confortée parfois par une labellisation et, souvent, par le rôle qu'y jouent les réseaux du commerce équitable : café ou cacao d'altitude, produits sur les versants chauds et humides des cordillères par de petites paysanneries, fruits tropicaux, eaux-de-vie comme le Pisco des vignobles des oasis du Sud péruvien, laine de vigogne de l'altiplano andin... Ailleurs ce sont des zones où le savoir-faire ancien d'une population - notamment dans le textile ou la céramique -, comme dans certaines régions d'Équateur ou du Pérou, peut être stimulé par le tourisme, voire complété et dynamisé par des options productives nouvelles. D'autres lieux, enfin, tirent bénéfice de l'exploitation touristique des ressources 
patrimoniales, historiques (monumentales) ou naturelles : ainsi des nombreux sites classés au patrimoine mondial de l'Unesco (du Machu Picchu aux îles Galápagos); de l'andinisme et des sports de montagne, dans les cordillères (volcans d'Équateur, Cordillère Blanche du Pérou, stations de montagne du Chili et de l'Argentine); et des foyers touristiques majeurs comme Cuzco ou Carthagène (destinations du tourisme national, continental et mondial) ou encore Cuenca ou Potosí.

Les espaces de conservation de la nature et de la biodiversité (parcs nationaux et zones protégées) constituent un quatrième type de périphéries, même s'ils se rattachent en partie aux zones de ressources patrimoniales naturelles précédemment évoquées. À cette différence près que le principe même de leur protection est censé les faire échapper aux flux du tourisme, en même temps que leur création répond souvent à une injonction de la culture et de la sensibilité écologique mondialisée, avec le souci de la sanctuarisation de ce qu'il reste de vie sauvage... Une situation non exempte de contradictions quand ces zones protégées sont habitées par des populations autochtones (dans les Andes et en Amazonie), ou quand elles sont soumises à d'importants flux touristiques originaires des pays du Nord (îles Galápagos).

Si les quatre types qui précèdent forment ensemble une espèce d'archipel complexe de lieux plus ou moins intégrés et différemment articulés à la mondialisation des échanges, le cinquième type correspond à des territoires, souvent beaucoup plus étendus, qui sont en marge de la modernité et de l'intégration aux dynamiques contemporaines. Il s'agit des replis reculés des cordillères, à l'écart des axes routiers importants et éloignés des villes. Ils semblent oubliés des pouvoirs publics, sinon des ONG. Certains observateurs les considèrent au mieux comme des conservatoires de traditions paysannes millénaires menacées de disparition, au pire comme des lieux de "stockage» de populations rurales - et souvent indiennes - qualifiées de «surnuméraires»... Ces zones de montagne, pour la plupart fondamentalement rurales et indiennes, forment d'amples poches de marginalisation et d'indigence. L'espace cultivable y est peu abondant, l'activité agricole demeure difficile du fait de conditions peu propices à la modernisation, et les paysanneries n'y ont pourtant jamais été aussi nombreuses qu'aujourd'hui, en dépit des flux migratoires qui ont conduit plusieurs générations de jeunes vers les terres chaudes des piémonts, vers les villes et, de plus en plus maintenant, vers les pays étrangers.

Un dernier type d'espaces périphériques occupe une place singulière. Il s'agit, le plus souvent, de fractions très étendues des territoires nationaux, situées notamment à l'est des Andes (savanes et forêts tropicales humides au nord, zones arides du Chaco et steppes de Patagonie, au sud). Très faiblement peuplées, hormis quelques rares îles urbaines (Leticia, Iquitos) et quelques enclaves de production (sites pétroliers surtout), ces régions sont peu ou mal contrôlées par les États et constituent l'essentiel des angles morts des territoires nationaux. Immensité, inaccessibilité relative, frontières internationales poreuses y ont favorisé l'établissement de sanctuaires des mouvements de guérillas (Colombie surtout, Pérou en mode résiduel) et, souvent associé à la présence combinée et conflictuelle de groupes armés et d'hommes de mains de notables corrompus, de zones d'activités (cultures illicites, laboratoires clandestins) et de trafics divers (armes, stupéfiants, contrebande) qui assurent une 
autre intégration à la mondialisation, mais côté antimonde. Une situation qui ne laisse pas d'inquiéter le Commandement Sud de l'armée des États-Unis pour lequel les "espaces vides» (Amazonie, Patagonie) et certaines "zones de non-droit» sont considérés comme une "nouvelle menace», qui pèse sur l'Amérique du Sud et compromet la sécurité de l'hémisphère, au même titre que le "terrorisme international», le "narcoterrorisme» - que l'absence de contrôle militaire et policier favorise -, et le "populisme radical». Et de pointer, parmi les zones sensibles, les frontières entre Colombie et Venezuela et Colombie et Équateur (Putumayo), la zone transfrontalière de Leticia - Tabatinga (entre Colombie, Brésil et Pérou) mais également la triple frontière entre Argentine, Brésil et Paraguay...

Quelles que soient les spécificités de chacun des pays andins (poids territorial ou démographique, diversification plus ou moins grande des activités...), la structuration de l'espace y présente donc nombre d'éléments de convergence. Des capitales très dynamiques, parfois élevées au rang de villes mondiales ou en train d'y parvenir, y dominent souvent sans partage l'ensemble du territoire national et sont le relais des donneurs d'ordres situés au Nord, étroitement liés à la Triade et à l'archipel mégalopolitain mondial. Les cellules du "monde à part» des enclaves introduisent dans leur environnement territorial des ruptures et des discontinuités économiques, sociales et spatiales souvent brutales. De grandes zones de production, intensivement mises en valeur, tirent parti de leur intégration ou de leur bonne articulation, à la fois aux marchés extérieurs et aux aires centrales des États. D'autres territoires, restreints le plus souvent, tirent profit de spécificités locales, alors que de vastes périphéries, territoires des cultures traditionnelles et des minorités ethnolinguistiques pour la plupart, sont en proie aux crises, au déclin, à l'abandon. De grands espaces montagneux, arides ou forestiers forment enfin de vastes angles morts des États. Les effets - d'intensité variable selon leur position par rapport aux centres - de l'intégration des pays andins au sein d'organisations supranationales et du système monde s'expriment donc dans un kaléidoscope de dynamiques territoriales, reflet de l'inégale internalisation d'une mondialisation qui développe les différences spatiales et les disparités sociales.

\section{Le monde andin dans la mondialisation}

Depuis le $\mathrm{xvI}^{\mathrm{e}}$ siècle, les grandes puissances n'ont cessé d'imposer leur marque aux espaces, aux sociétés et aux économies du sous-continent latino-américain. L'Espagne a d'abord largement exploité les populations et les richesses de l'espace andin pendant la période coloniale. Au XIx ${ }^{e}$ siècle, la Grande-Bretagne et la France, ainsi que les États-Unis dans une moindre mesure à partir de l'affirmation de la doctrine Monroe en 1823, ont pris le relais en s'appuyant sur les élites cosmopolites et les groupes dominants. Ces grandes puissances extrarégionales protégeaient leurs intérêts stratégiques (contrôle des passages et des détroits), politiques (intervention en sous-main dans les conflits opposant les pays andins) et économiques (investissements dans l'exploitation des ressources minières et agropastorales). À partir de la première guerre mondiale, les États-Unis ont éclipsé les puissances européennes. Au début du Xxi ${ }^{e}$ siècle, leur domination est parfois battue en brèche par les grands pays asiatiques. 
Depuis 2003, la tendance générale du PIB est à la croissance dans tous les pays andins : cependant, cette progression repose essentiellement sur les exportations de produits du secteur primaire et, à l'exception du Venezuela peu attractif en ce domaine, sur les investissements directs étrangers. Ces moteurs ont certes un effet d'entraînement sur le reste de l'économie et sur la consommation des ménages, mais ils sont fragiles, et la bonne tenue des agrégats macroéconomiques a un impact limité sur l'éradication de la pauvreté, la réduction des inégalités et le renforcement de l'intégration régionale. Malgré de timides avancées, celle-ci piétine et parvient difficilement à tirer parti de ce terrain de jeu mondial où s'affrontent les puissants blocs de l'Accord de libre-échange nord-américain (ALENA-NAFTA) et de l'Union européenne ainsi que les nouvelles puissances asiatiques.

\section{La permanence d'un fort degré d'extraversion économique}

Face aux résultats mitigés du modèle d'industrialisation par substitution des importations mis en place après la deuxième guerre mondiale et partiellement abandonné dans les années 1970, le néolibéralisme est devenu le nouveau modèle économique à suivre à partir des années 1980. Les politiques qui en sont l'expression et qui s'appuient sur le retrait de l'État, la privatisation des services publics, les réformes financières, l'ouverture des marchés et l'entrée massive des capitaux étrangers semblent porter des fruits, mais elles reposent sur des bases instables (exploitation intensive de ressources non renouvelables, volatilité des cours mondiaux) et leur coût social est élevé. Dans le contexte historique continental, ce retour à l'exploitation des produits primaires (extraction minière, mise en valeur des gisements d'hydrocarbures, productions agropastorales), qui s'accompagne parfois d'un processus de désindustrialisation, évoque le retour à un système d'exploitation de type colonial, remis au goût du jour.

Le degré d'extraversion économique des pays andins est cependant différencié. Certains pays disposent de ressources qui leur permettent de se placer dans le peloton de tête des pays producteurs et/ ou exportateurs au niveau mondial. Le Chili est ainsi le $1^{\mathrm{er}}$ producteur de cuivre, le $2^{\mathrm{e}}$ de molybdène et le $5^{\mathrm{e}} \mathrm{d}^{\prime}$ argent (les produits miniers représentent plus de la moitié de ses exportations et le cuivre près de $90 \%$ de ses exportations minières); le Pérou est le $1^{\mathrm{er}}$ producteur $\mathrm{d}^{\prime}$ argent, le $3^{\mathrm{e}}$ de cuivre, d'étain et de zinc, le $4^{e}$ de plomb et de molybdène et le $5^{e} \mathrm{~d}^{\prime}$ or ; la Colombie est le $1^{\text {er }}$ producteur d'émeraudes, etc. ${ }^{14}$. Si les ressources pétrolières et gazières sont importantes en Équateur, en Bolivie, en Colombie, au Pérou et en Argentine, le Venezuela, $10^{\mathrm{e}}$ producteur et $8^{\mathrm{e}}$ exportateur de pétrole, est le pays andin le mieux

14 - Le Chili, qui produit $37 \%$ du cuivre mondial (Chuquicamata est la plus grande mine de cuivre à ciel ouvert du monde), extrait aussi du fer, du manganèse, de l'or, de l'argent... Le Pérou produit $16 \%$ de l'argent mondial et $10 \%$ du plomb. La Colombie assure $60 \%$ de la production mondiale d'émeraudes. Les données relatives à l'extraction et l'exportation des ressources naturelles (2004 ou 2005) sont tirées des sites Internet de l'Energy Information Administration et de l'USGS; celles qui traitent des productions agricoles, des exportations, des clients et fournisseurs des pays andins en 2005 proviennent des sites Internet de la FAO et des missions économiques des ambassades de France dans les pays andins. 
doté. Les productions agroalimentaires, celles de la pêche et de l'aquaculture, sont l'un des secteurs moteurs des exportations de l'Argentine (la viande bovine, les céréales et le soja - presque exclusivement transgénique - représentent environ $50 \%$ des ventes à l'étranger), de la Colombie ( $2^{\mathrm{e}}$ exportateur mondial de café et de fleurs coupées) et de l'Équateur ( $1^{\mathrm{er}}$ exportateur de bananes et de farine de poisson et $8^{\mathrm{e}}$ de fèves de cacao, $1^{\mathrm{er}}$ producteur de thon et $2^{\mathrm{e}}$ de crevettes d'élevage). Le niveau d'extraversion économique des pays andins varie aussi en fonction de la diversification des économies nationales : le tissu industriel de l'Argentine, de la Colombie, et dans une moindre mesure du Pérou, est plus dense que celui de la Bolivie ou du Venezuela. Cependant, les industries dynamiques et les services supérieurs sont aussi très dépendants des investissements étrangers (secteur automobile en Colombie et en Argentine; industries agroalimentaires; téléphonie où la compagnie espagnole Telefónica investit massivement; secteur bancaire au Chili, constitué de vingt-six banques, dont douze étrangères qui détiennent 39 \% des encours).

Le PNB des pays andins, indicateur de leur poids aux niveaux régional et mondial, varie dans un rapport de 1 à 20 (9 milliards en Bolivie et 173 en Argentine, en 2005). Si le taux de croissance annuel du PNB est élevé dans les sept pays - supérieur à $10 \%$ en raison de l'envolée du prix du pétrole, il est spectaculaire au Venezuela depuis 2004 - il repose en grande partie sur la progression des cours des matières premières. La forte participation des produits miniers et des hydrocarbures à la constitution du PNB montre la fragilité de cette croissance, en grande partie exogène (fig. 7). Trois groupes se différencient cependant: dans le premier, les matières premières non agricoles entrent pour plus d'un quart dans la formation du PNB (Venezuela $34 \%$ et Chili $26 \%$ ); l'Équateur (21\%) est en situation intermédiaire entre ce premier groupe et le deuxième, qui réunit la Bolivie et la Colombie, où les produits miniers, le pétrole et le gaz participent à la formation du PNB pour moins d'un quart; enfin, le Pérou et l'Argentine forment un troisième groupe où la part des matières premières non agricoles intervient pour moins de $15 \%$ dans la formation du PNB (si elle ne dépasse pas $12 \%$ en Argentine, où la filière agricole et alimentaire constitue $14 \%$ du PIB et reste le secteur moteur des ventes à l'étranger, elle est cependant supérieure à la moyenne des pays sud-américains qui s'établit à $8 \%$ ).

La structure du commerce extérieur permet d'esquisser une typologie qui s'appuie à la fois sur le volume et la valeur des échanges et sur le taux de couverture - équilibre ou déséquilibre des importations par rapport aux exportations - (fig. 8) : elle est similaire à la classification établie à partir des indicateurs démographiques et des niveaux de développement humain. Le Venezuela, l'Argentine et le Chili s'y caractérisent par l'intensité de leurs échanges avec l'extérieur ${ }^{15}$. Si le solde des échanges est excédentaire dans les trois pays, le taux de couverture vénézuélien est supérieur à $200 \%$ (solde positif de 30 milliards de dollars contre 13 milliards en Argentine) alors que celui du Chili atteint «à peine» 140 \%. Le deuxième groupe rassemble la

15 - Le rapport entre les exportations vénézuéliennes (56,8 milliards de dollars en 2005) et boliviennes (2,7 milliards) est de 1 à 21 ; les importations du Venezuela (27,3 milliards) et de l'Argentine (27,2 milliards) sont 12 fois supérieures à celles de la Bolivie (2,3 milliards). 


\section{FIGURE 7 : MATIÈRES PREMIÈreS ET PRODUITS MANUFACTURÉS DANS LES PAYS ANDINS EN 2004}

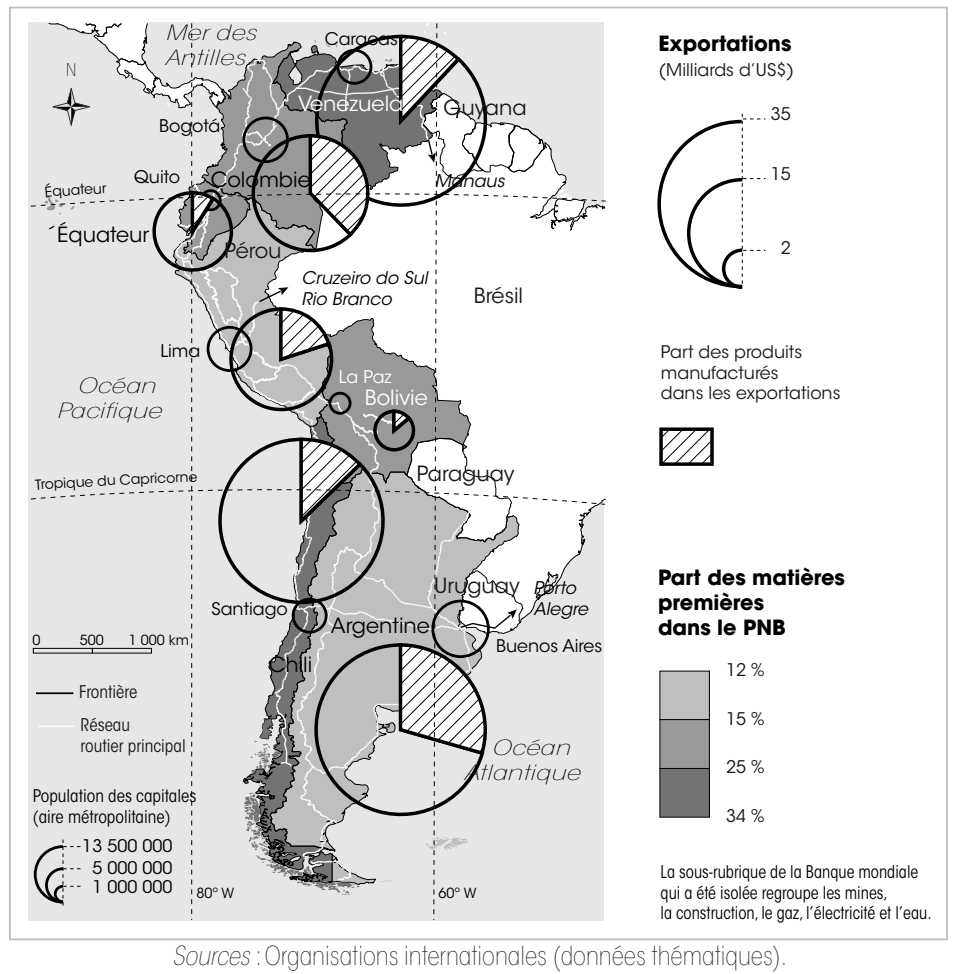

Colombie, le Pérou et l'Équateur, par ordre décroissant des échanges ${ }^{16}$. Le Pérou se démarque cependant des deux autres pays par un taux de couverture supérieur à 130 \% alors que celui de la Colombie et de l'Équateur n'est que très légèrement excédentaire. Enfin, le dernier groupe est constitué de la seule Bolivie au faible volume d'échanges (moins de 3 milliards de dollars aux importations et aux exportations) et au taux de couverture un peu inférieur à $120 \%$. Si en 2005 le solde de la balance commerciale était positif dans les sept pays, l'excédent dégagé était très variable : il s'échelonnait entre un peu plus de 200 millions de dollars en Équateur et près de 30 milliards au Venezuela ${ }^{17}$.

16 - L'Équateur est en position intermédiaire entre le deuxième et le troisième groupe, constitué d'un seul pays. Ses exportations et ses importations atteignent à peine 10 milliards de dollars, alors que les exportations du Pérou et de la Colombie ainsi que les importations colombiennes dépassent 16 milliards de dollars.

17 - L'augmentation du prix du cours du pétrole d'un dollar entraîne une augmentation des recettes vénézuéliennes de 960 millions de dollars et un accroissement du PIB de 0,4\%. En raison de la flambée des cours des matières premières, les exportations tendent généralement à stagner ou à augmenter légèrement en volume alors qu'elles progressent fortement en valeur (elles ont presque doublé au Chili entre 2003 et 2005, passant de 21 milliards de dollars à près de 40 milliards; au Venezuela, pendant la même période, elles sont passées de 28 à près de 67 milliards de dollars en 2006, année au cours de laquelle le pétrole et les services pétroliers ont constitué $88 \%$ des exportations totales - le prix moyen annuel du baril de pétrole Brent est passé de 28,9 dollars US en 2003 à 54,4 dollars US en 2005 et à 65,1 dollars US en 2006. 
Figure 8 : Le COMMERCE EXTÉRIEUR DES PAYS ANDINS EN 2005

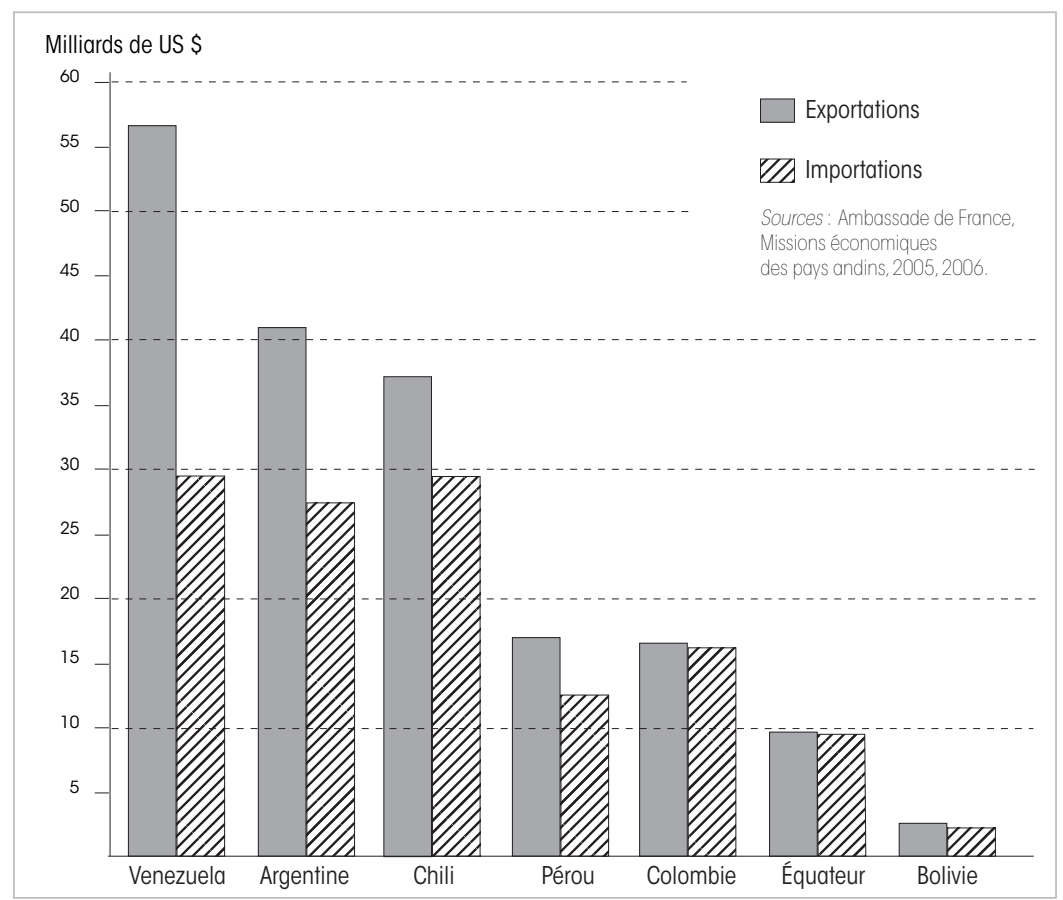

Il est surprenant que la majorité de la population vénézuélienne vive toujours dans des conditions qui ne reflètent pas la richesse de la nation : en effet, malgré les efforts accomplis par l'État et les politiques sociales mises en ouvre en faveur des catégories sociales les plus défavorisées (les dépenses engagées au titre des Missions de la Révolution bolivarienne représenteraient entre $4 \%$ et $7 \%$ du PIB), la pauvreté n'a pas été résorbée et les inégalités se sont accentuées.

L'analyse de la part des produits manufacturés dans les exportations, autre indicateur de l'extraversion économique des pays andins dont l'intensité varie en fonction de leur structure productive, corrobore la typologie établie à partir de la structure du commerce extérieur (fig. 7). La Colombie et l'Argentine se détachent, grâce à leur base industrielle, les produits manufacturés représentant respectivement $38 \%$ et $29 \%$ de la valeur totale des exportations. Leur part est inférieure à $15 \%$ dans les autres pays andins, à l'exception du Pérou où les produits manufacturés entrent pour $20 \%$ dans les exportations.

Si la structure des échanges rend compte de la forte dépendance des économies andines à l'égard du marché mondial et de la vulnérabilité de leur croissance, l'étude des principaux partenaires commerciaux permet d'approcher le degré d'intégration économique des pays andins au sein des deux organisations économiques régionales : la Communauté andine (CAN), qui rassemble la Bolivie, la Colombie, l'Équateur et le Pérou, et le Marché commun du sud (Mercosur), qui 
réunit l'Argentine, le Brésil, le Paraguay, l'Uruguay et le Venezuela (ce dernier a quitté la CAN en avril 2006).

\section{Difficultés et aléas du processus d'intégration régionale}

Si les échanges commerciaux progressent entre les États andins, notamment dans le cadre de chacune des deux organisations régionales dont ils sont membres, ils restent relativement limités. Les États-Unis sont en effet le client et le fournisseur dominant, voire quasi exclusif, des pays des Andes du Nord, tandis que dans les autres pays, où leur poids dans les échanges est moins écrasant, ils restent dans le trio de tête des pays importateurs et exportateurs (fig. 9) ${ }^{18}$. Au Venezuela, en Colombie et en Équateur, ils absorbent au moins $40 \%$ des exportations et le rapport entre le premier et le deuxième client est supérieur à 4 ; leur domination dans les importations du Venezuela et de la Colombie est moins marquée (entre 20 et 30 \%) mais le rapport entre les deux premiers pays fournisseurs y est proche de 4 . Bien que les relations politiques et diplomatiques entre les gouvernements vénézuélien et états-unien soient tendues, voire hostiles, le Venezuela demeure le pays andin le plus dépendant du marché des États-Unis - et de l'Accord de libre-échange nord-américain (ALENA), dans une moindre mesure; les États-Unis sont en effet le débouché de $60 \%$ des exportations vénézuéliennes (le Canada, en se classant deuxième, en absorbe moins de $3 \%$ ) et assurent près de $25 \%$ des importations du pays (le Brésil étant en deuxième position avec un peu plus de $8 \%$ des ventes). En Bolivie, le rôle dominant revient au Brésil, grande puissance du Mercosur, au premier rang des pays importateurs. L'Argentine est le seul des pays andins à avoir pour premier partenaire commercial un pays de son organisation régionale d'appartenance : le Brésil, avec lequel elle rivalise pour le leadership du Mercosur. Avec des rapports entre pays clients et fournisseurs moins déséquilibrés, le Chili et l'Argentine, pour les exportations, le Chili, l'Équateur et la Bolivie, pour les importations, sont moins dépendants d'un seul marché.

Les échanges entre les États membres du Mercosur ont progressé au début du $\mathrm{xxI}^{\mathrm{e}}$ siècle. L'Argentine, qui réalisait en 2000 plus d'un quart de ses exportations et un cinquième de ses importations avec les pays du Mercosur, y réalise près de $30 \%$ de ses ventes et $40 \%$ de ses achats en 2005. Les échanges du Chili avec les États du Mercosur sont importants, principalement aux importations, mais ceux avec l’Union européenne sont supérieurs.

En revanche, le commerce entre les pays de la Communauté andine (CAN) reste souvent modeste, à l'exception de l'Équateur qui réalise $15 \%$ de ses exportations et $23 \%$ de ses importations avec les autres États membres. Au Pérou, les importations en provenance des pays de la CAN devancent les achats aux membres de l'Union européene (19\% contre $12 \%)$, mais l'ordre s'inverse aux exportations (respectivement $6 \%$ et $17 \%$ ); en Colombie, l'Union a devancé la CAN tant aux exportations qu'aux importations. En Bolivie, qui réalise plus de $10 \%$ de ses exportations et importations

18 - En 2006, les États-Unis étaient le premier client et/ou fournisseur de cinq pays andins (au Chili, ils ont déplacé l'Argentine au second rang des pays importateurs). 
avec les États membres de la CAN, c'est le Mercosur qui l'emporte largement, avec près de la moitié des ventes et des achats boliviens à l'extérieur assurés par le Brésil et l'Argentine. À partir de 2007, le volume des échanges internes aux pays de la CAN sera affecté par le départ du Venezuela, dont la Colombie est le deuxième partenaire ( $8 \%$ des importations et $10 \%$ en 2006) loin derrière les États-Unis, et qui a quitté cette organisation - trop néo-libérale pour Hugo Chávez - pour rejoindre le Mercosur ${ }^{19}$.

Les deux marchés communs régionaux réunissent des pays de structures économiques très différentes. Ils ont des poids démographique, économique et politique très contrastés et leurs rapports de forces sont donc inégaux. La CAN, réduite désormais à quatre pays andins, rassemble 100 millions d'habitants, alors que le Mercosur, renforcé par l'adhésion du Venezuela, en réunit 260 millions sur presque toute la façade atlantique de l'Amérique du Sud. Le PNB des États de la CAN s'élève à 220 milliards de dollars alors que celui du Mercosur est plus de quatre fois supérieur (970 milliards de dollars). Enfin, alors que les pays de la CAN sont seulement des puissances régionales moyennes ou faibles, le Mercosur abrite les deux grandes puissances sud-américaines, dont l'influence s'étend bien au-delà de l'Amérique latine ${ }^{20}$.

Les deux blocs économiques régionaux doivent faire face à des rivalités et à des tensions internes qui expliquent en partie la lenteur et les aléas d'une intégration difficile, même si les outils politiques, législatifs et judiciaires de la CAN sont plus performants que ceux du Mercosur. Depuis le début des années 2000, les États membres de la Communauté andine tentent de multiplier les accords bilatéraux, principalement avec les États-Unis, afin de les substituer aux accords préférentiels qui les lient aux mêmes États-Unis, mais dans le cadre de l'Andean Trade Preferential Drug Eradication Act (ATPDE). La Colombie et le Pérou ont ainsi signé, en 2006, un traité de libre commerce (TLC), non encore ratifié, avec les États-Unis, et l'Équateur s'engageait sur la même voie avant les élections qui ont porté Rafael Correa à la présidence en novembre 2006. Les quatre pays de la CAN sont aussi membres associés au Mercosur. Au sein de ce dernier, le Brésil et l'Argentine se disputent la suprématie dans l'organisation régionale tandis que les relations sont tendues entre l'Argentine et l'Uruguay, à la suite du projet d'installation de deux papeteries à capitaux européens sur le fleuve Uruguay, à proximité de la frontière entre les deux pays, l'Argentine accusant l'Uruguay de vouloir exporter les effluents pollués de ces usines en territoire argentin. Le Chili, qui n'est membre ni de la CAN ni du Mercosur, auquel il est cependant associé, a signé de nombreux traités de libre-échange (dont un TLC avec les États-Unis, en 2003) et regarde vers les pays asiatiques.

Les recompositions économiques et l'évolution des rapports de force au niveau international incitent les deux organisations régionales à multiplier les contacts avec les grandes puissances et avec les blocs économiques majeurs, ALENA, Union

19 - En 2005, le Venezuela a absorbé 30 \% des exportations intracommunautaires.

20 - Le Pacte andin, constitué de cinq pays (avec le Chili sans le Venezuela, puis sans le Chili et avec le Venezuela) a été créé en 1969; il a donné naissance à la Communauté andine des nations (CAN) en 1988. Le Mercosur, créé en 1991 et entré en vigueur en 1995, est contemporain de l'ALENA (signé en 1992) que le Mexique a intégré en 1994. 
Figure 9 - PrinCIPAUX CLIENTS et fournISSEURS DES PAYS ANDINS EN 2005



européenne et Coopération économique Asie-Pacifique (APEC), tout en essayant de mettre en place une Communauté sud-américaine des nations (CSAN) visant à regrouper la CAN et le Mercosur. C'est en décembre 2004 que le sommet de Cuzco avait permis un rapprochement du Mercosur, de la CAN et du Chili et la signature de l'acte fondateur de la CSAN, les «États-Unis d'Amérique du Sud». En 2007, les relations entre les organisations régionales économiques et politiques sont complexes. Face à l'échec de la constitution de la Zone de libre-échange des Amériques (ZLEA), les États-Unis s'appuient sur la signature de traités de commerce bilatéraux (TLC) destinés à élargir l'ALENA et censés permettre de lutter contre les avancées de l'Union européenne, d'une part, et celles de l'Alternative bolivarienne pour les Amériques (ALBA), initiative d'Hugo Chávez, d'autre part. Contreproposition à dimensions sociales à une ZLEA exclusivement économique, l'ALBA 
réunit le Venezuela, la Bolivie et plusieurs îles antillaises, dont Cuba; l'Équateur et le Nicaragua sont intéressés par cet axe La Havane-Caracas-La Paz, dont les membres signent des Traités de commerce des peuples (TCP contre TLC) ${ }^{21}$. Alors que le Mercosur a signé un accord avec l'Union européenne dès 1998, un accord entre la CAN et l'UE, qui se réuniront à Lima en mai 2008 à l'occasion du Ve sommet entre les deux organisations, pourrait voir le jour dans les années à venir. Les pays andins se sont aussi ouverts sur l'Asie puisque le Chili et le Pérou font partie de l'APEC depuis 1992 et 1998 et que le Chili a signé un traité de libre-échange avec la Corée du Sud en 2004. La zone Asie-Pacifique, au potentiel indiscutable, absorbe d'ailleurs plus d'un quart des exportations chiliennes et péruviennes.

\section{Conclusion : changements politiques, continuité économique?}

Depuis la fin des années 1990, on assiste à l'essoufflement du modèle politique, porteur des thèses d'un néolibéralisme économique censé résoudre les nombreux problèmes des pays latino-américains. Déçues et désabusées par la corruption, l'inégale redistribution des richesses et l'accroissement des inégalités, les populations ont perdu confiance dans les partis "traditionnels» en crise et recherchent de nouvelles voies, d'où la montée en puissance ou les éclats de mouvements sociaux (populaires, associatifs, ethniques), et l'émergence de nouveaux acteurs de la société civile. Cette évolution s'accompagne d'un "virage à gauche» dans les urnes qui n'exclut pas les risques de dérive populiste, comme l'Amérique latine en a déjà connu dans son histoire. Avec six élections présidentielles dans les pays andins, l'année 2006 a été riche en événements qui ont souvent confirmé ces tendances. Mais de Michelle Bachelet, élue en mars au Chili, à Rafael Correa, élu en Équateur en octobre et à Hugo Chávez, réélu au Venezuela en décembre, les options idéologiques et les orientations économiques sont variées, dans une région andine où se côtoient et s'opposent des populations et des espaces intégrés au système monde et les populations des vastes territoires exclus de la croissance et du développement. Si le changement politique est progressiste, au flux de cette tendance à l'installation de gouvernements allant du centre-gauche à la gauche populiste - à l'exception notable de la Colombie de Álvaro Uribe, sinon du Pérou de Alan García - le cap économique suivi reste le plus souvent néo-libéral, tant sont fortes les contraintes du système économique mondial dominant. Et si on peut s'attendre à la mise en œuvre de politiques visant à une certaine amélioration du sort des plus pauvres, elles risquent de trouver leur limite dans la difficile réforme d'un système économique dont la dynamique se nourrit des différences de potentiels régionaux et de la permanence d'inégalités sociospatiales.

21 - La mise en place d'une coopération énergétique régionale pourrait devenir un accélérateur de l'intégration : création d'un consortium pétrolier sud-américain (Petroamérica); projet de construction d'un gazoduc de $10000 \mathrm{~km}$ reliant le Venezuela à l'Argentine par la Bolivie permettant d'approvisionner les pays du Cône sud. 


\section{Bibliographie}

- Beaverstock J.V., R. G. Smith et P. J. TAYlor, "A Roster of World Cities", Cities, 16(6), 1999, p. 445-458 (http://www.lboro.ac.uk/ gawc/rb/rb5.html).

- Blanquer J.-M. et P. Zagefka (dir.), Amérique latine 2005, Paris, La Documentation française et Institut des hautes études de l'Amérique latine (IHEAL), 2005, 205 p.

- Central Intelligence Agency (CIA), The World Factbook 2007, 2007 (https://www.cia. gov/cia/publications/factbook/index.html).

- COMISIÓn ECONÓMICA PARA AmÉRICA LATINA (CEPAL), Anuario estadístico de América Latina y el Caribe 2005, Santiago du Chili, CEPAL, 2006, 438 p. (http://www.eclac.org/cgibin/getProd.asp? xml=/publicaciones/xml/0/26530/ P26530.xml\&xsl=/deype/tpl/p9f.xsl\&base=/tpl/ top-bottom.xslt).

- DABÈne O. (dir.), Atlas de l'Amérique latine. Violences, démocratie participative et promesses de développement, Paris, Autrement, 2006, $80 \mathrm{p}$.

- Dollfus O., La mondialisation, Paris, Presses de Sciences Po, 1997, 167 p.

- Bataillon C., J.-P. Deler et H. Théry (dir.), Amérique latine, 1991, rééd. Paris, Belin-Reclus, Géographie universelle, 2000, 480 p.

- Dureau F., V. Gouëset et É. Mesclier (dir.), Géographies de l'Amérique latine, Rennes, Presses universitaires de Rennes, 2006, 375 p.

- ENERGy InFormation AdMinistration (EIA), Bases statistiques relatives aux productions énergétiques, Washington, EIA, 2007 (http://www. eia.doe.gov/).

- Foucher M., Fronts et frontières. Un tour du monde géopolitique, Paris, Fayard, 1991, 610 p., cartes.

- Fonds des NATIONS UNIES POUR LA POPULATION (UNFPA), État de la population mondiale 2006. Vers l'espoir: les femmes et la migration internationale, New York, UNFPA, 2006, 107 p. (http://www.unfpa.org/swp/index_fre.html).

- Food and Agricultural Organization of THE UnITED STATES (FAO), The statistics division, Rome, FAO, 2007 (http://www.fao.org/es/ess/ toptrade/trade.asp).
- Hardy S. et L. Medina-Nicolas (dir.), L'Amérique latine, Paris, Éditions du temps, coll. "Questions de géographie», 2005, 285 p.

- La Documentation fRAnÇAise, Dossier "Amérique latine», Questions internationales, $\mathrm{n}^{\circ} 18,2006,127 \mathrm{p}$.

- L'état du monde. Annuaire économique géopolitique mondial 2006, Paris, La Découverte, 2005, 666 p.

- Naciones UnidAs-Comisión ECONÓmica PARA América Latina (CEPAL), Anuario estadístico de América Latina y el Caribe 2005, Santiago du Chili, Nations unies-CEPAL, 2006, 438 p. (http://www.eclac.org/cgibin/getProd.asp?xml=/ publicaciones/xml/0/26530/P26530.xml\&xsl=/ deype/tpl/p9f.xsl\&base=/tpl/top-bottom.xslt).

- NATIONS Unies-OfFice CONTRE LA DROGUE ET LE CRIME, 2006 Rapport mondial sur les drogues, Vienne, UNDOC, 2007, vol. 1 et 2 (http://www. unodc.org/pdf/WDR_2006/wdr06_french_vol1. www.pdf, http://www.unodc.org/pdf/WDR_2006/ wdr06_french_vol2.www.pdf).

- Population Reference Bureau (PRB), 2006 World Population Data Sheet, Washington, PRB, 2006, 12 p. (http://www.prb.org/pdf06/ 06WorldDataSheet.pdf).

- Sassen S., La ville globale, Paris, Descartes \& Cie, 1996, 533 p.

- The World Bank Group, 06 World Development Indicators, Washington, The World Bank Group, 2006, n.p. (http://devdata.worldbank. org/wdi2006/contents/cover.htm).

- United Nations Development Programme (UNDP), Human Development Report 2006. Beyond Scarcity: Power, Poverty and the Global Water Crisis, New York, UNDP, 2006, 422 p. (http://hdr.undp.org/hdr2006/).

- United States GeOlogical Survey (USGS), Mineral information, Washington, USGS, 2007 (http:// minerals.usgs.gov/minerals/pubs/commodity/).

- Veltz P., Mondialisation villes et territoires. L'économie d'archipel, Paris, Presses universitaires de France, 1997, 262 p.

- Velut V. (dir.), L'Amérique latine, Paris, SEDES-CNED, 2006, 365 p. 